\title{
Energy dispersion in turbulent jets. Part 2. A robust model for unsteady jets
}

\author{
John Craske ${ }^{1, \dagger}$ and Maarten van Reeuwijk ${ }^{1}$ \\ ${ }^{1}$ Department of Civil and Environmental Engineering, Imperial College London, London SW7 2AZ, UK
}

(Received 24 March 2014; revised 24 September 2014; accepted 14 November 2014;

first published online 18 December 2014)

In this paper we develop an integral model for an unsteady turbulent jet that incorporates longitudinal dispersion of two distinct types. The model accounts for the difference in the rate at which momentum and energy are advected (type I dispersion) and for the local deformation of velocity profiles that occurs in the vicinity of a sudden change in the momentum flux (type II dispersion). We adapt the description of dispersion in pipe flow by Taylor (Proc. R. Soc. Lond. A, vol. 219, 1953, pp. 186-203) to develop a dispersion closure for the longitudinal transportation of energy in unsteady jets. We compare our model's predictions to results from direct numerical simulation and find a good agreement. The model described in this paper is robust and can be solved numerically using a simple central differencing scheme. Using the assumption that the longitudinal velocity profile in a jet has an approximately Gaussian form, we show that unsteady jets remain approximately straight-sided when their source area is fixed. Straight-sidedness provides an algebraic means of reducing the order of the governing equations and leads to a simple advection-dispersion relation. The physical process responsible for straight-sidedness is type I dispersion, which, in addition to determining the local response of the area of the jet, determines the growth rate of source perturbations. In this regard the Gaussian profile has the special feature of ensuring straight-sidedness and being insensitive to source perturbations. Profiles that are more peaked than the Gaussian profile attenuate perturbations and, following an increase (decrease) in the source momentum flux, lead to a local decrease (increase) in the area of the jet. Conversely, profiles that are flatter than the Gaussian amplify perturbations and lead to a local increase (decrease) in the area of the jet.

Key words: jets, mixing, turbulence modelling

\section{Introduction}

The objective of this paper is to develop a robust model describing the evolution of the integral properties of an unsteady turbulent jet. Whilst there are several unsteady jet models based on extensions of classical plume theory (e.g. Delichatsios 1979; Yu 1990; Scase et al. 2006, which will be discussed in detail in $\$ 2.2$ ), it has recently been shown, for the more general case of plumes, that most of these are ill-posed (Scase \& Hewitt 2012). Specifically, the models are ill-posed because they do not

†Email address for correspondence: john.craske07@imperial.ac.uk 
account for longitudinal interaction in the jet and therefore admit the development of unbounded short-wave modes. One exception to this case is the model developed by Scase \& Hewitt (2012), who introduced a longitudinal mixing term based on an eddy diffusivity closure, which renders the system of equations well-posed.

In Part 1 (Craske \& van Reeuwijk 2015) dispersion was identified as a core process governing the dynamics of unsteady jets. We distinguished as dispersive all transport processes resulting from non-uniform velocity profiles rather than uniform top-hat velocity profiles. Realistic velocity profiles were found to give rise to two distinct types of dispersion.

(a) Type I dispersion results from the fact that laterally non-uniform, albeit self-similar, profiles of longitudinal velocity cause energy to be advected faster than momentum.

(b) Type II dispersion results from a departure from self-similarity. In particular, laterally non-uniform velocity profiles acting over longitudinal gradients can cause a departure from self-similarity.

Type II dispersion, rather than longitudinal turbulence transport, was shown to be the primary agent for longitudinal mixing in Part 1. Type II dispersion is associated with shear-flow dispersion, a form of longitudinal mixing first identified by Taylor (1953) in relation to the dispersion of soluble matter in a pipe flow. Shear-flow dispersion was subsequently formalised by Aris (1956), whose formulation in terms of longitudinal moments revealed that the effects of shear flow dispersion appear in sum with those of longitudinal diffusion. In the field of transport processes in rivers and estuaries, Taylor's ideas have received widespread attention (see e.g. Fischer et al. 1979); for a comprehensive review of the mathematical techniques employed in such applications the reader is referred to Fischer (1973) and Chatwin \& Allen (1985). To date, the application of dispersion theory has been focused on bounded flows rather than unbounded free-shear flows.

More recently, Landel, Caulfield \& Woods (2012) addressed how cross-stream variations in velocity affect both advection and dispersion in a quasi-two-dimensional jet. They proposed a one-dimensional model that employs Prandtl's classical mixing length theory and exploits the fact that the scales of the eddies responsible for mixing are determined by the local characteristic scales of the jet at a particular longitudinal location. A similar reasoning was used to justify the inclusion of turbulent mixing via a second-order derivative in the unsteady plume model of Scase \& Hewitt (2012). Indeed, in Scase \& Hewitt (2012) it was shown that the inclusion of turbulent mixing eliminates the growth of short-wave modes that render the original model of Scase et al. (2006) ill-posed. However, for jets, the observations we reported in Part 1 suggest that type II dispersion rather than turbulence transport per se provides the dominant source of longitudinal mixing.

Both type I and type II dispersion account for the longitudinal interaction of lateral slices of a jet, which is a feature that is absent from top-hat models of jets and plumes (Scase, Aspden \& Caulfield 2009; Scase \& Hewitt 2012). However, it is type I, rather than type II, dispersion that represents the leading-order effect of laterally non-uniform velocity profiles. Consequently, type I dispersion has a fundamental influence on the way in which quantities such as momentum, energy and area are distributed over the longitudinal dimension of an unsteady jet. More precisely, type I dispersion determines the relative rates with which momentum and energy are advected, the separation of characteristic curves and the extent to which the area of an unsteady jet departs from its steady-state behaviour. 
In this paper we capitalise on the momentum-energy framework presented in Part 1 to develop a robust and accurate integral model that describes the dominant transport processes of unsteady jets. A notable difference between this approach and that of previous unsteady plume models is that the use of the momentum-energy framework ensures consistency between the governing equations for area, momentum and the energy of the mean flow, regardless of the profile of velocity that is assumed. In order to obtain a closed system of equations that can be used in a prognostic capacity, a dispersion closure is developed that captures the propagation (type I dispersion) and the spreading rate (type II dispersion) of disturbances in the jet.

The work is organised as follows. Following a summary of the momentum-energy framework in $\S 2.1$, in $\S 2.2$ we review several existing unsteady plume models and show that, from an energetics perspective, none of the ill-posed unsteady plume models employ a realistic assumption regarding the radial profile of longitudinal velocity. We show in $\S 2.3$ that in the absence of buoyancy the mixing term suggested by Scase \& Hewitt (2012) does not conserve momentum, because it introduces non-physical source terms in both the momentum and mean energy equation. In $\S 3$ we develop a dispersion closure for jets based on the observations reported in Part 1 and the analysis conducted by Taylor $(1953,1954)$. We compare predictions obtained using our model to both direct numerical simulation (DNS) results and the regularised unsteady plume model of Scase \& Hewitt (2012) in $\S 4$. In $\$ 5$ we show theoretically that under certain conditions Gaussian jets remain approximately straight-sided and use this result to propose several possible simplifications of the model developed in $\S 3$. Finally, in $\S 6$ we analyse the governing equations in the absence of mixing to demonstrate the effect that type I dispersion has on the growth of perturbations and the behaviour of a jet's velocity and radius in the vicinity of a step change in the momentum flux.

\section{Unsteady jet models}

\subsection{Exact formulation}

Here we consider the equations governing the motion of fluid induced by a source of volume and specific momentum flux $\left(Q_{0}, M_{0}\right)$ located at $z=0$. As discussed in detail in Part 1, we focus on the evolution of integral quantities of the flow, whose governing equations are obtained by integrating pointwise three-dimensional conservation equations over a horizontal disk centred on the axis of the jet. The radius of the disk is $r_{d}(z, t)$, which is defined according to $\bar{w}\left(r_{d}, z, t\right)=\epsilon \bar{w}(0, z, t)$, where $\epsilon \ll 1$ and $\bar{w}$ is the ensemble-average longitudinal velocity. Hence $r_{d}$ encompasses most of the longitudinal motion comprising the jet and we neglect longitudinal fluxes of momentum and energy entering the jet from the ambient. Integration over the horizontal disk of pointwise conservation equations for volume, momentum and the energy associated with the mean flow results in the following system (Part 1):

$$
\begin{gathered}
\frac{1}{\gamma_{g}} \frac{\partial A_{m}}{\partial t}+\frac{\partial Q_{m}}{\partial z}=2 \alpha M_{m}^{1 / 2}, \\
\frac{\partial Q_{m}}{\partial t}+\frac{\partial\left(\beta_{g} M_{m}\right)}{\partial z}=0, \\
\frac{\partial M_{m}}{\partial t}+\frac{\partial}{\partial z}\left(\gamma_{g} \frac{M_{m}^{2}}{Q_{m}}\right)=\delta_{g} \frac{M_{m}^{5 / 2}}{Q_{m}^{2}} .
\end{gathered}
$$


Here, $A_{m} \equiv Q_{m}^{2} / M_{m}$ is the characteristic area of the jet, and the dependent variables are the mean volume flux $Q_{m}$ and the mean specific momentum flux $M_{m}$, hereafter referred to as the volume flux and the momentum flux, respectively:

$$
Q_{m} \equiv 2 \int_{0}^{r_{d}(z, t)} \bar{w} r \mathrm{~d} r, \quad M_{m} \equiv 2 \int_{0}^{r_{d}(z, t)} \bar{w}^{2} r \mathrm{~d} r .
$$

Given the form of (2.2) and (2.3) and the definitions (2.4a,b), it will occasionally prove useful to regard $Q_{m}$ and $M_{m}$ not as fluxes, but as the integrals of (specific) momentum and (specific) energy of the mean flow at a given height. The parameter $\alpha$ is the classical entrainment coefficient (see e.g. Morton, Taylor \& Turner 1956; Turner 1986). Length and velocity scales for the flow are defined as $r_{m} \equiv Q_{m} / M_{m}^{1 / 2}$ and $w_{m} \equiv M_{m} / Q_{m}$, respectively. Equations (2.1)-(2.3) comprise an extension to unsteady problems of the integral equations posed by Priestley \& Ball (1955) and later by Kaminski, Tait \& Carazzo (2005), though in the absence of buoyancy. In particular, the only assumptions made in our derivation are that the Reynolds number $R e \gg 1$ and that longitudinal fluxes entering the jet from the ambient at $r=r_{d}$ are negligible.

In (2.1)-(2.3), we use Greek letters to denote dimensionless parameters, corresponding to unknown fluxes and turbulence production terms. Specifically, we define the dimensionless gross flux of momentum

$$
\beta_{g} \equiv 1+\underbrace{\frac{2}{M_{m}} \int_{0}^{r_{d}(z, t)} \overline{w^{\prime 2}} r \mathrm{~d} r}_{\beta_{f}}+\underbrace{\frac{2}{M_{m}} \int_{0}^{r_{d}(z, t)}\left(\bar{p}-p_{d}\right) r \mathrm{~d} r}_{\beta_{p}}
$$

which includes longitudinal turbulent transport $\beta_{f}$ and the pressure integral $\beta_{p}$. Here $w^{\prime}$ represents turbulent fluctuations in the longitudinal velocity field. The pressure $\bar{p}$ and its boundary value $p_{d} \equiv \bar{p}\left(r_{d}, z, t\right)$ are both relative to a hydrostatic pressure and hence $p_{d} \approx 0$ in the cases considered here. It should be noted that due to the longitudinal development of turbulent transport in the near field, the source momentum flux $M_{0}$ is typically higher than the mean momentum flux $M_{m}$. Similarly, the dimensionless gross energy flux is defined according to

$$
\gamma_{g}=\underbrace{\frac{2 Q_{m}}{M_{m}^{2}} \int_{0}^{r_{d}(z, t)} \bar{w}^{3} r \mathrm{~d} r}_{\gamma_{m}}+\underbrace{\frac{4 Q_{m}}{M_{m}^{2}} \int_{0}^{r_{d}(z, t)} \bar{w} \overline{w^{2}} r \mathrm{~d} r}_{\gamma_{f}}+\underbrace{\frac{4 Q_{m}}{M_{m}^{2}} \int_{0}^{r_{d}(z, t)}\left(\bar{p}-p_{d}\right) \bar{w} r \mathrm{~d} r}_{\gamma_{p}},
$$

where $\gamma_{m}$ corresponds to transport by the mean flow, $\gamma_{f}$ to turbulent transport and $\gamma_{p}$ to pressure work. Finally, we define the dimensionless turbulence production and pressure redistribution terms according to

$$
\delta_{g} \equiv \underbrace{\frac{4 Q_{m}^{2}}{M_{m}^{5 / 2}} \int_{0}^{r_{d}(z, t)} \overline{u^{\prime} w^{\prime}} \frac{\partial \bar{w}}{\partial r} r \mathrm{~d} r}_{\delta_{m}}+\underbrace{\frac{4 Q_{m}^{2}}{M_{m}^{5 / 2}} \int_{0}^{r_{d}(z, t)} \frac{\partial \bar{w}}{w^{\prime 2}} \frac{\partial \bar{w}}{\partial z} r \mathrm{~d} r}_{\delta_{f}}+\underbrace{\frac{4 Q_{m}^{2}}{M_{m}^{5 / 2}} \int_{0}^{r_{d}(z, t)} \bar{p} \frac{\partial \bar{w}}{\partial z} r \mathrm{~d} r}_{\delta_{p}},
$$

which account for turbulence production due to shear, longitudinal fluctuations and pressure redistribution, respectively. The dimensionless parameters defined in (2.5)-(2.7) allow one to describe the flow in one dimension, without making an assumption about the magnitude or shape of the unknown radial profiles, in 


\begin{tabular}{lccrrc} 
& TH & $\mathrm{G}$ & \multicolumn{1}{c}{$\mathrm{H}$} & \multicolumn{1}{c}{$\mathrm{L}$} & PL93 \\
$\alpha$ & $\alpha_{0}$ & $\alpha_{0}$ & $0.065 \pm 0.001$ & $0.069 \pm 0.002$ & 0.082 \\
$\beta_{f}$ & 0.000 & 0.000 & $0.157 \pm 0.001$ & $0.160 \pm 0.004$ & 0.187 \\
$\beta_{p}$ & 0.000 & 0.000 & $-0.088 \pm 0.001$ & $-0.091 \pm 0.002$ & - \\
$\beta_{g}$ & 1.000 & 1.000 & $1.068 \pm 0.001$ & $1.069 \pm 0.002$ & - \\
$\gamma_{m}$ & 1.000 & 1.333 & $1.303 \pm 0.003$ & $1.298 \pm 0.005$ & 1.305 \\
$\gamma_{f}$ & 0.000 & 0.000 & $0.284 \pm 0.003$ & $0.292 \pm 0.006$ & 0.354 \\
$\gamma_{p}$ & 0.000 & 0.000 & $-0.171 \pm 0.001$ & $-0.185 \pm 0.006$ & - \\
$\gamma_{g}$ & 1.000 & 1.333 & $1.416 \pm 0.006$ & $1.404 \pm 0.006$ & - \\
$\delta_{m}$ & $-2 \alpha_{0}$ & $-8 \alpha_{0} / 3$ & $-0.187 \pm 0.003$ & $-0.195 \pm 0.006$ & -0.201 \\
$\delta_{f}$ & 0.000 & 0.000 & $0.006 \pm 0.000$ & $0.007 \pm 0.001$ & - \\
$\delta_{p}$ & 0.000 & 0.000 & $-0.002 \pm 0.000$ & $-0.003 \pm 0.000$ & -
\end{tabular}

TABLE 1. The dimensionless parameters of a steady jet. Here TH refers to top-hat, $G$ to Gaussian, $\mathrm{H}$ and $\mathrm{L}$ to simulations of a jet with Reynolds number $2 M_{0} / v=6810$ and 4815, respectively, where $M_{0}$ is the source momentum flux, and PL93 to the experimental data of Panchapakesan \& Lumley (1993). The parameter $\alpha_{0}$ is the steady-state entrainment coefficient. The values displayed in the columns beneath $\mathrm{H}$ and $\mathrm{L}$ are given to within one standard deviation. For further details see Part 1.

terms of the primary unknowns $Q_{m}$ and $M_{m}$. Table 1 displays the value of these parameters obtained from the DNS of two steady-state turbulent jets, compared with the experimental observations of Panchapakesan \& Lumley (1993).

Although the momentum-energy approach of Priestley \& Ball (1955) predates the volume-momentum formulation of Morton et al. (1956), the latter emerged as the standard model for plumes (see e.g. Hunt \& van den Bremer 2011). Only recently, when Kaminski et al. (2005) showed that a momentum-energy formulation allows one to establish an expression for the entrainment coefficient in terms of turbulence production and several profile constants has there been a revival of interest in the momentum-energy approach. In the intervening years, Fox (1970) also recognised that consideration of the mean flow energetics allows one to understand properties of turbulent entrainment that are not accessible at the level of volume conservation alone. In the analysis of unsteady jets, and by implication unsteady plumes, employing a mean flow energy equation (2.3) brings further advantages.

Pertaining to both type I and type II dispersion, the dimensionless energy flux

$$
\gamma_{m} \equiv 2 \frac{Q_{m}}{M_{m}^{2}} \int_{0}^{r_{d}(z, t)} \bar{w}^{3} r \mathrm{~d} r
$$

has a dominant role in both steady and unsteady jets. Notable is the fact that when $\bar{w}$ is assumed to have a top-hat form, then $\gamma_{m}=1$, yet when $\bar{w}$ is assumed to have a Gaussian form $\gamma_{m}=4 / 3$. The difference of $1 / 3$ between these values greatly exceeds the contributions $\beta_{f}, \beta_{p}$ and $\gamma_{f}$, due to turbulent transport (see table 1 and Part 1). Perhaps more importantly, $\gamma_{m}$ provides a means of accounting for both type $\mathrm{I}$ and type II dispersion in terms of the mean flow: type I dispersion is determined by the steady-state value of $\gamma_{m}$, whilst type II dispersion is determined by the response of $\gamma_{m}$ to local changes in $Q_{m}, M_{m}$ and/or their derivatives. Therefore, to develop a simple model for unsteady jets that accounts for dispersion,we focus on the role of $\gamma_{m}$ and 
set $\gamma_{g}=\gamma_{m}, \beta_{g}=1, \delta_{g}=\delta_{m}$ in (2.1)-(2.3):

$$
\begin{gathered}
\frac{1}{\gamma_{m}} \frac{\partial A_{m}}{\partial t}+\frac{\partial Q_{m}}{\partial z}=2 \alpha M_{m}^{1 / 2}, \\
\frac{\partial Q_{m}}{\partial t}+\frac{\partial M_{m}}{\partial z}=0, \\
\frac{\partial M_{m}}{\partial t}+\frac{\partial}{\partial z}\left(\gamma_{m} \frac{M_{m}^{2}}{Q_{m}}\right)=\delta_{m} \frac{M_{m}^{5 / 2}}{Q_{m}^{2}} .
\end{gathered}
$$

Equations (2.9)-(2.11) focus on the transport of momentum and energy by the mean flow rather than turbulence. The implication of (2.10) and (2.11) is that there is no longitudinal mixing of momentum $Q_{m}$. In addition, the propagation speed of disturbances in the jet depends only on $M_{m}, Q_{m}$ and the local value of $\gamma_{m}$, which in turn is determined by an assumed mean velocity profile. In reality, the presence of turbulent transport modifies the propagation speed and, if there is a local change in the relative intensity of turbulence $\beta_{f}$, can result in a discernible redistribution of momentum. In spite of these simplifications we will see that turbulence continues to play a notable role via $\delta_{m}$ in the final term of (2.11), which represents the conversion of energy in the mean flow to turbulence kinetic energy.

The system (2.9)-(2.11) appears to be over-determined, because in the original threedimensional equations the mean energy equation is obtained from a combination of the momentum equation and the continuity equation. Thus, in (2.9)-(2.11) there are two independent equations and a third, whose consistency with the others depends on the closure that is employed for $\alpha$. In this way it is possible to obtain the expression

$$
\alpha \equiv \underbrace{-\frac{\delta_{m}}{2 \gamma_{m}}}_{\alpha_{\text {prod }}}+\underbrace{\frac{Q_{m}}{M_{m}^{3 / 2}}\left(1-\frac{1}{\gamma_{m}}\right) \frac{\partial M_{m}}{\partial z}}_{\alpha_{\text {disp } 1}}+\underbrace{\frac{Q_{m}}{2 M_{m}^{1 / 2} \frac{\partial \ln \gamma_{m}}{\partial z}}}_{\alpha_{d i s p 2}},
$$

which ensures consistency among the three equations. In (2.12) $\alpha_{\text {prod }}$ accounts for the ratio of turbulence production and mean energy flux; $\alpha_{d i s p 1}$ accounts for type I dispersion and is equal to zero for top-hat jets, for which $\gamma_{m}=1$; and $\alpha_{\text {disp } 2}$ accounts for type II dispersion and is zero when the jet is everywhere self-similar. The decomposition (2.12) is similar to that employed by Kaminski et al. (2005) to quantify the effect that buoyancy in plumes has on entrainment in a steady state. For further details the reader is referred to Part 1.

\subsection{Existing unsteady jet models}

In this section we compare the formulation (2.9)-(2.11), which makes no assumption regarding the radial profile of longitudinal velocity, to the unsteady plume models of Delichatsios (1979), Yu (1990) and Scase et al. (2006). For the purposes of comparison, we will assume that $\gamma_{m}$ is constant, which is equivalent to assuming that the velocity profiles remain self-similar and that type II dispersion is equal to zero. By adopting the traditional perspective of area and momentum conservation, in the absence of buoyancy the models are captured by

$$
\begin{gathered}
\frac{1}{\gamma_{m}} \frac{\partial}{\partial t} \frac{Q_{m}^{2}}{M_{m}}+\frac{\partial Q_{m}}{\partial z}=2 \alpha_{0} M_{m}^{1 / 2}+c \frac{2 Q_{m}}{M_{m}}\left(1-\frac{1}{\gamma_{m}}\right) \frac{\partial M_{m}}{\partial z}, \\
\frac{\partial Q_{m}}{\partial t}+\frac{\partial M_{m}}{\partial z}=0 .
\end{gathered}
$$




$\begin{array}{lcc} & \gamma_{m} & c \\ \text { Delichatsios (1979) } & 2 & 0 \\ \text { Yu (1990) } & 1 & 1 \\ \text { Scase } \text { et al. (2006) } & 1 & 1 \\ \text { Gaussian } & 4 / 3 & 1 \\ \text { Top-hat } & 1 & 1\end{array}$

TABLE 2. Comparison of unsteady plume models applied to a jet. For further details see (2.13) and (2.14).

Here, $\alpha_{0}=-\delta_{m} /\left(2 \gamma_{m}\right)$, for constant $\delta_{m}$, is the steady-state entrainment coefficient and we have introduced $c$ to account for an inconsistency between a model's equations and the exact area equation (2.9). Thus, the values $c=0$ or $c=1$ indicate whether the model is inconsistent or consistent, respectively, when considering mean energy and momentum conservation.

The models of Delichatsios (1979) and Yu (1990) are given by $\left(\gamma_{m}, c\right)=(2,0)$ and $\left(\gamma_{m}, c\right)=(1,1)$. Both claim to assume Gaussian profiles for the longitudinal velocity. However, the comparison shows that in neither of these models does one find the exact theoretical value $\gamma_{m}=4 / 3$, which can be obtained by assuming a Gaussian distribution in (2.8). Instead, in Delichatsios (1979), whose equations are ostensibly based on the Lagrangian formulation employed by Middleton (1975) but are not derived explicitly, one finds $\gamma_{m}=2$. A testament to the ambiguity and consequent difficulty surrounding the unsteady area equation is that $\mathrm{Yu}$ (1990), also assuming a Gaussian profile, find $\gamma_{m}=1$. However, as pointed out by Scase \& Hewitt (2012), the derivation of $\mathrm{Yu}$ (1990) is questionable in the light of the divergent integral appearing in their mass conservation equation. With $\left(\gamma_{m}, c\right)=(1,1)$, the model of $\mathrm{Yu}(1990)$ is consistent with (2.13); the model of Delichatsios (1979), on the other hand, is inconsistent in this respect. In conclusion, it appears that neither of these models can be referred to as genuine Gaussian models. In particular, the model of Yu (1990) has the characteristics of a top-hat model, which explains why Scase \& Hewitt (2012) identify it as having a similar dynamic response to the model of Scase et al. (2006). Indeed, the model of Scase et al. (2006), which is derived explicitly using top-hat profiles, is fully consistent with (2.13) and (2.14). Noteworthy, however, is the fact that whilst the equations of Scase et al. (2006) are only valid for uniform (top-hat) profiles, the system (2.9)-(2.11) is valid for arbitrary velocity profiles.

Not included in table 2, which shows a comparison of the unsteady plume models, is the model of Vul'fson \& Borodin (2001), which is unique in stipulating that the plume remains conical, or straight-sided, independently of time-dependent changes that occur at its source; although Scase \& Hewitt (2012) remark that the appropriateness of such an assumption is an open question. In this regard we have discovered that Gaussian jets, even if their momentum flux is subjected to a sudden change, have the remarkable property of remaining straight-sided, provided that their source area is fixed. This feature of the jet equations will be discussed in greater detail in $\S 5$, and, more generally, in $\S 6$ we illustrate the effect that any underlying velocity profile has on both the local area and the downstream growth rate of perturbations. Therefore for jets, compared to the models listed in table 2, the model of Vul'fson \& Borodin (2001) provides the only system that is fully consistent with the assumption of a Gaussian profile. 
With the exception of the refinement suggested by Scase \& Hewitt (2012), which will be discussed in the following section, none of the models above accounts for the possibility of longitudinal mixing, i.e. for type II dispersion or turbulent transport. However, clear from the results presented in Part 1 is the fact that the longitudinal spreading of fronts is significant and should therefore be included.

\subsection{The regularisation of ill-posed models}

In Scase \& Hewitt (2012) it was pointed out that the unsteady plume models of Delichatsios (1979), Yu (1990) and Scase et al. (2006) are ill-posed in their admission of the unbounded growth of short-wave modes. It is not a coincidence, given the discussion of the previous section, that the only model identified as being well-posed was that of Vul'fson \& Borodin (2001). To render the models well-posed it was recommended (Scase \& Hewitt 2012) that a diffusion term be introduced in the momentum equation. In this section we identify several problems with the diffusion term that was proposed, and suggest that a more appropriate closure involves the mixing of energy in the mean flow via shear-flow dispersion.

In the absence of buoyancy, the area and momentum equations proposed in Scase \& Hewitt (2012) are

$$
\begin{gathered}
\frac{\partial}{\partial t}\left(\frac{Q_{m}^{2}}{M_{m}}\right)+\frac{\partial Q_{m}}{\partial z}=2 \alpha_{0} M_{m}^{1 / 2}, \\
\frac{\partial Q_{m}}{\partial t}+\frac{\partial M_{m}}{\partial z}=\frac{\beta_{1}}{2 \alpha_{0}} \frac{Q_{m}^{2}}{M_{m}} \frac{\partial}{\partial z}\left[M_{m}^{1 / 2} \frac{\partial}{\partial z}\left(\frac{M_{m}}{Q_{m}}\right)\right],
\end{gathered}
$$

where $\beta_{1}$ is a dimensionless constant. Unlike the original system of equations, the system (2.15) and (2.16) does not admit steady-state solutions of the form $Q_{m}=2 \alpha_{0} M_{m}^{1 / 2} z$, i.e. solutions in which the jet radius $r_{m} \equiv Q_{m} / M_{m}^{1 / 2}=2 \alpha_{0} z$. This behaviour is in contrast to that of plumes, for which the model was derived, in which the classical steady-state radius $r_{m}=(6 / 5) \alpha_{0} z$ is unaffected by the additional mixing term. To understand the cause of the problem, it is useful to rearrange (2.16) as

$$
\frac{\partial Q_{m}}{\partial t}+\frac{\partial}{\partial z}\left[M_{m}-\frac{\beta_{1}}{2 \alpha_{0}} \frac{Q_{m}^{2}}{M_{m}^{1 / 2}} \frac{\partial}{\partial z}\left(\frac{M_{m}}{Q_{m}}\right)\right]=-\frac{\beta_{1}}{2 \alpha_{0}} M_{m}^{1 / 2} \frac{\partial}{\partial z}\left(\frac{Q_{m}^{2}}{M_{m}}\right) \frac{\partial}{\partial z}\left(\frac{M_{m}}{Q_{m}}\right) .
$$

The closure can now be understood as a combination of a modified flux of momentum on the left-hand side and a source term on the right-hand side. Thus, due to the source term, a steady jet does not conserve momentum using the closure proposed by Scase \& Hewitt (2012). In fact, a steady-state power-law solution to the system (2.15) and (2.16) can be found:

$$
\begin{gathered}
M_{m}=M_{0}\left(1-\frac{z}{z_{v}}\right)^{\Phi}, \\
Q_{m}=-\frac{2 \alpha_{0} M_{0}^{1 / 2} z_{v}}{\Phi / 2+1}\left(1-\frac{z}{z_{v}}\right)^{\Phi / 2+1},
\end{gathered}
$$

where $z_{v}$ is the location of a virtual source of zero momentum flux and zero volume flux, $z_{v} \equiv-(\Phi / 2+1) Q_{0} /\left(2 \alpha_{0} M_{0}^{1 / 2}\right)$, and $M_{0}$ and $Q_{0}$ are positive quantities denoting 
the mean momentum flux and volume flux at $z=0$, respectively. The exponent in (2.18) and (2.19) is found to be

$$
\Phi \equiv \frac{2 \beta_{1}-\sqrt{8 \beta_{1}+1}+1}{\beta_{1}-1} .
$$

In contrast to plumes, in jets the diffusion term has the undesirable effect of modifying the classical power-law dependences, because jets do not contain a forcing term on the right-hand side of their momentum equation. This illustrates the fact that modification of the plume equations is best tackled at the simpler level of the jet equations, which place a greater restriction on the possible forms that can be introduced.

Of further interest is the fact that (2.15) and (2.16) imply a mean energy balance

$$
\frac{\partial M_{m}}{\partial t}+\frac{\partial}{\partial z}\left[\frac{M_{m}^{2}}{Q_{m}}-\frac{\beta_{1}}{2 \alpha_{0}} \frac{Q_{m}^{2}}{M_{m}^{1 / 2}} \frac{\partial}{\partial z}\left(\frac{M_{m}^{2}}{Q_{m}^{2}}\right)\right]=-2 \alpha_{0} \frac{M_{m}^{5 / 2}}{Q_{m}^{2}}-\frac{\beta_{1}}{\alpha_{0}} M_{m}^{1 / 2}\left(\frac{\partial Q_{m}}{\partial z}\right) \frac{\partial}{\partial z}\left(\frac{M_{m}}{Q_{m}}\right),
$$

which illustrates that a modification of the momentum and/or area equation manifests itself in the mean energy equation. We argue that it is more beneficial to account for longitudinal mixing in the mean energy equation directly, in a manner that is physically realistic.

\section{A dispersion closure for turbulent jets}

In this section we develop a dispersion closure for unsteady jets by restricting our attention to the transport of a passive scalar quantity. In particular, we consider the flux $F_{m}$ of the scalar quantity $\bar{c}=c_{m}(z) g(\eta, z)$ in a known steady velocity field $\bar{w}=$ $w_{m}(z) f_{0}(\eta)$, where $\eta=r / r_{m}$. We will assume that the upper limit of all radial integrals $r_{d} \rightarrow \infty$. In $\S 3.1$ we consider type I dispersion by examining a self-similar steady state. In $\S 3.2$ we consider departures from this steady state and apply the analysis of Taylor (1953) to obtain an expression for type II dispersion.

\subsection{Type I dispersion}

One of the main advantages of employing the mean energy equation is that it contains nonlinear terms whose value depends on the correlation of mean flow properties over the radius. To appreciate the leading-order effect that this correlation has on the flux $F_{m}$, we will consider a self-similar, steady state in which the function $g=g_{0}(\eta)$ and $f=f_{0}(\eta)$. Indeed, $f_{0}$ and $g_{0}$ can be defined such that their integrals are equal to unity:

$$
2 \int_{0}^{\infty} f_{0}(\eta) \eta \mathrm{d} \eta=2 \int_{0}^{\infty} g_{0}(\eta) \eta \mathrm{d} \eta=1
$$

When $f_{0}$ and $g_{0}$ differ from uniform distributions of the same width, their correlation might prevent us from equating the top-hat product $w_{m} c_{m} r_{m}^{2}$ with $F_{m}$ :

$$
F_{m} \equiv 2 w_{m} c_{m} r_{m}^{2} \int_{0}^{\infty} f_{0}(\eta) g_{0}(\eta) \eta \mathrm{d} \eta \neq w_{m} c_{m} r_{m}^{2}
$$

in general. Thus spatial averaging introduces additional unknown correlations in the same way that they are introduced at a fixed location by a temporal or ensemble average. If the scalar profile $g_{0}$ is narrower than a non-uniform monotonically 
decreasing distribution $f_{0}$, then $F_{m}>w_{m} c_{m} r_{m}^{2}$, due to the concentration of the scalar in regions where $\bar{w}$ is relatively large. To see this, assume that $f_{0}(\eta)=2 \exp \left(-2 \eta^{2}\right)$ and

$$
g_{0}(\eta)=\frac{2}{\mu^{2}} \exp \left(-2 \frac{\eta^{2}}{\mu^{2}}\right) .
$$

Here $\mu r_{m}$ is the lateral length scale associated with the radial dependence of $\bar{c}$. Using (3.3), the scalar flux can be determined exactly:

$$
F_{m} \equiv 2 w_{m} c_{m} r_{m}^{2} \int_{0}^{\infty} \frac{4}{\mu^{2}} \exp \left[-2 \eta^{2}\left(1+\frac{1}{\mu^{2}}\right)\right] \eta \mathrm{d} \eta=\theta_{0} w_{m} c_{m} r_{m}^{2},
$$

where, for the particular case of Gaussian profiles $f_{0}$ and $g_{0}$,

$$
\theta_{0}=\frac{2}{1+\mu^{2}} \text {. }
$$

The parameter $\theta_{0}$ appeared in the unsteady plume model of Delichatsios (1979) to account for the difference in lateral spread between radial profiles of buoyancy and momentum. In Part 1 we identified those profile effects that are associated with the steady state as giving rise to type I dispersion, and showed that they were responsible for a difference in the rates at which energy and momentum are transported in a jet. A Gaussian approximation to the steady energy distribution in a jet shows that $f_{0}^{2}(\eta)=$ $4 \exp \left(-4 \eta^{2}\right)$. Hence $\mu^{2}=1 / 2$ and $\theta_{0}$, which in the case of energy we denote as $\gamma_{0}$, is equal to $4 / 3$.

\subsection{Type II dispersion}

A difficulty associated with type II dispersion, unlike type I, is that it cannot be investigated without perturbing a jet from a steady self-similar state. In general the longitudinal divergence of the scalar flux $F_{m} \equiv \theta_{m} w_{m} c_{m} r_{m}^{2}$ is

$$
\frac{\partial F_{m}}{\partial z}=\theta_{m} \frac{\partial\left(w_{m} c_{m} r_{m}^{2}\right)}{\partial z}+w_{m} c_{m} r_{m}^{2} \frac{\partial \theta_{m}}{\partial z} .
$$

Type II dispersion occurs when $\theta_{m}$ is not constant, and therefore results in the second term on the right-hand side of (3.6) being non-zero.

The classical view of dispersion originates from analysis undertaken by Taylor (1953) of the dispersion of soluble matter in a pipe flow. Taylor demonstrated that in the vicinity of a step reduction in the mean concentration of the solute there emerges a region in which the otherwise uniform solute concentration is modified by a concentration that varies in the radial direction. Responsible for the deformation in the mean concentration profile is the radial variation of longitudinal velocity, because material located on the centreline of the pipe is transported faster than material located near the walls.

Taylor reasoned that for relatively large times the local deformation in the concentration would satisfy a balance between longitudinal advection and radial diffusion:

$$
\frac{1}{r} \frac{\partial}{\partial r}\left(v_{T} r \frac{\partial \tilde{c}}{\partial r}\right)=\tilde{w} \frac{\partial c_{m}}{\partial z}
$$

where $c_{m}(z, t)$ is the mean cross-section concentration and $\tilde{c}(r, z, t)$ is the deformation in the concentration profile, such that the total mean concentration $\bar{c}=c_{m}+\tilde{c}$. 
The velocity $\tilde{w}$ is relative to a frame of reference moving with the cross-section mean velocity $w_{m}$. Equation (3.7) implies that the deformation $\tilde{c}$ has the same radial dependence for all $z$ and $t$, and has an amplitude that is proportional to $v_{T}^{-1} \partial_{z} c_{m}$, for a constant eddy viscosity $v_{T}$.

Using (3.7) to determine $\tilde{c}$, the scalar flux $F_{m}$ can be obtained as the sum of a steady-state component and a component arising from the correlation of $\tilde{c}$ with $\tilde{w}$ :

$$
F_{m} \equiv \theta_{0} w_{m} c_{m} r_{m}^{2}+2 \int_{0}^{\infty} \tilde{w} \tilde{c} r \mathrm{~d} r .
$$

For pipe flow, whose boundaries provide a physical radial scale, we will regard $r_{m}$ as the pipe radius. In contrast, jets are unbounded in the radial direction and it is necessary to appeal to moments of the velocity profile in order to determine a suitable radial scale, according to $r_{m} \equiv Q_{m} / M_{m}^{1 / 2}$. Indeed, in order for (3.8) to be applicable to jets, we integrate to infinity, but recognise that for pipe flow the integral has an upper limit of $r_{m}$. In addition, for pipe flow, the self-similar steady-state scalar profile is uniform and $\theta_{0}=1$. The main observation to be made from (3.8) is that the second term on the right-hand side will, in accordance with (3.7), depend on $\partial_{z} c_{m}$. Therefore, the flux $F_{m}$ will vary according to the longitudinal gradient of the mean concentration.

Fundamental to both dispersion in pipe flow and dispersion in jets is the idea that changes in the mean scalar concentration in the longitudinal direction can cause a local departure from self-similarity over the lateral dimension. The aim here is to apply Taylor's treatment of dispersion in pipes to type II dispersion in jets. Our emphasis is on obtaining a simple and intuitive analogue to Taylor dispersion that focuses on the dominant physics. We do not intend to obtain a complicated model whose relation to classical Taylor dispersion is lost. Hence, we follow a heuristic approach and recast the balance (3.7) in a form that can be applied to jets.

The fundamental difference between dispersion in pipe flow and dispersion in jets is that in the latter the mean steady-state concentration $c_{m 0}(z)$ varies in the longitudinal direction. To recast the mean scalar concentration in a jet in a form that is comparable to the mean scalar concentration in a pipe, it is convenient to examine the ratio

$$
\mathscr{C}_{m}(z, t) \equiv \frac{c_{m}}{c_{m 0}},
$$

which is constant for all $z$ in the case of a steady flow, regardless of the way in which $c_{m 0}$ depends on $z$. Note that for pipe flow $c_{m 0}$ is constant and $c_{m}$ can be replaced with $\mathscr{C}_{m}$ in (3.7) without approximation.

In addition, a steady-state scalar distribution in a jet will have a non-uniform radial dependence, which will not necessarily have compact support. Therefore, to describe the steady-state concentration we use the similarity function $g_{0}(\eta)$, introduced in $\S 3.1$. It is also convenient to introduce the function $g_{1}(\eta)$ to describe departures from the basic radial dependence of $g_{0}$. We can therefore express the dimensionless concentration field to leading order according to

$$
\frac{\bar{c}(r, z, t)}{c_{m 0}}=\mathscr{C}_{m}\left(g_{0}+L_{1} g_{1}\right),
$$

where $L_{1}$ determines the relative amplitude of the departures from the steady-state profile $g_{0}$, and is therefore assumed to be independent of $\eta$. In general it is convenient to define $\tilde{w}$ relative to a coordinate moving with the steady velocity $\theta_{0} w_{m}$, which 
means that $\tilde{w} \equiv w_{m}\left(f_{0}-\theta_{0}\right)$, where $f_{0}(\eta)$ is the self-similar velocity profile introduced in $\S 3.1$.

We will assume that the eddy viscosity $v_{T}$ is constant and equal to $\alpha_{0} w_{m} r_{m} / 3$. For jets, $\alpha_{0} w_{m} r_{m} / 3$ corresponds to an integral estimation of $v_{T}$ in which $\alpha_{0}$ is the steadystate entrainment coefficient (Part 1). Consequently, we can express Taylor's dispersion balance (3.7) in dimensionless form according to

$$
\frac{L_{1}}{\eta} \frac{\mathrm{d}}{\mathrm{d} \eta}\left(\eta \frac{\mathrm{d} g_{1}}{\mathrm{~d} \eta}\right)=-g_{0}\left(\theta_{0}-f_{0}\right)\left[\frac{3 r_{m}}{\alpha_{0}} \frac{1}{\mathscr{C}_{m}} \frac{\partial \mathscr{C}_{m}}{\partial z}\right] .
$$

A noteworthy property of the right-hand side of (3.11) is that it is not affected by a linear rescaling of the steady-state concentration: $c_{m 0} \mapsto a c_{m 0}$. This property is consistent with the view that the physics of the problem should not depend on the particular steady-state scalar concentration that is used in (3.9) to nondimensionalise $c_{m}$.

Because $L_{1}$ is responsible for all amplitude variations in $z$ and $t$, it follows that, to within a multiplicative constant,

$$
L_{1}=-\frac{3 r_{m}}{\alpha_{0}} \frac{1}{\mathscr{C}_{m}} \frac{\partial \mathscr{C}_{m}}{\partial z} .
$$

In this way the form of the leading-order departure from similarity $g_{1}$ is determined by the geometric properties of the steady-state velocity and concentration profiles, and is independent of $z$ and $t$. However, since we are concerned with integral quantities, it is not necessary to know the precise form of $g_{1}$. Indeed, due to their conical geometry, we suspect that for jets $g_{1}$ satisfies a slightly different ordinary differential equation in $\eta$ to that which is implied by (3.11). Nevertheless, from an integral perspective it is the dependence of $L_{1}$ on the dimensionless longitudinal gradient of the mean concentration $\mathscr{C}_{m}$, expressed in (3.12), that is of particular interest.

In analogy with $\theta_{0}$, we can now define another geometric parameter, $\theta_{1}$, to characterise the scalar flux arising from the departure from the basic steady-state similarity:

$$
\theta_{1} \equiv 2 \int_{0}^{\infty} f_{0} g_{1} \eta \mathrm{d} \eta
$$

The correlation of the perturbation profile $g_{1}$ with $f_{0}$ determines how the total dimensionless scalar flux depends on gradients in the longitudinal direction:

$$
\theta_{m}=\theta_{0}+L_{1} \theta_{1} .
$$

The dimensionless flux $\theta_{m}$ consists of the sum of a steady component $\theta_{0}$ and an unsteady component $L_{1} \theta_{1}$. Evident from (3.12) is that the contribution from the latter depends on the extent to which longitudinal gradients $\partial_{z} c_{m}$ deviate from their steady-state value. The constant geometric parameter $\theta_{1}$, which depends on $f_{0}$ and $g_{1}$, determines the sensitivity of the dimensionless flux $\theta_{m}$ to the departure from similarity that is quantified by $L_{1}$.

\subsection{Closure of $\gamma_{m}$}

Equation (3.14) comprises the dispersion closure advocated in this paper. This closure will now be applied to the dimensionless energy flux $\gamma_{m}$. We assume that, like the 
scalar flux $\theta_{m}$, the dimensionless energy flux $\gamma_{m}$ in a jet can be decomposed into a steady component $\gamma_{0}$ and an unsteady component $L_{1} \gamma_{1}$ :

$$
\gamma_{m}=\gamma_{0}+L_{1} \gamma_{1}
$$

where, like $\theta_{1}$, the constant geometric parameter $\gamma_{1}$ can in principle be determined from the steady self-similar velocity profile $f_{0}$ and the deformation profile $f_{1}$, which correspond to $g_{0}$ and $g_{1}$ in $\S 3.2$.

To determine $L_{1}$ we focus on the ratio $w_{m} / w_{m 0}$ in place of $\mathscr{C}_{m} \equiv c_{m} / c_{m 0}$ in (3.12). In addition, we define the local steady-state mean velocity $w_{m 0}$ so that $\partial_{z} w_{m 0}=-2 \alpha w_{m 0} / r_{m}$ and (3.12) becomes

$$
L_{1}=-\frac{3 r_{m}}{\alpha_{0}}\left(\frac{2 \alpha_{0}}{r_{m}}+\frac{1}{w_{m}} \frac{\partial w_{m}}{\partial z}\right) .
$$

In terms of integral quantities, the dimensionless energy flux (3.15) can therefore be expressed as

$$
\gamma_{m}=\gamma_{0}-6\left[1+\frac{Q_{m}^{2}}{2 \alpha_{0} M_{m}^{3 / 2}} \frac{\partial}{\partial z}\left(\frac{M_{m}}{Q_{m}}\right)\right] \gamma_{1} .
$$

The parameters $\gamma_{0}$ and $\gamma_{1}$ require calibration before the model can be used. Although it is possible to obtain values of $\gamma_{0}$ and $\gamma_{1}$ based on the geometry of the steady-state velocity profile alone, a more convenient and robust approach is to derive their values directly from observation. In Part 1 unsteady jets were produced by imposing an instantaneous step change up and down in the source momentum flux $M_{0}$. In the steady state we found that $\gamma_{m}=1.3$. For the unsteady jets, we estimated a dimensionless dispersion coefficient, $D_{e} / M_{m}^{* 1 / 2}=0.22$, by comparing self-similar profiles of $M_{m}(z, t)$ to a solution of an advection-dispersion equation for the mean energy. In $\S 5.2$ of this paper we will show that under certain assumptions $D_{e} / M_{m}^{* 1 / 2}=3 \gamma_{1} /\left(2 \alpha_{0}\right)$, which, when $\alpha_{0}=0.07$, provides a reasonable first estimation $\gamma_{1}=0.01$. For further detail regarding the relationship between $\gamma_{1}$ and $D_{e}$ the reader is referred to $\S 5.2$ of the present work. In addition, we note that $\gamma_{0}$ is equal to the steady-state value of $\gamma_{m}$ and therefore set

$$
\gamma_{0}=1.3, \quad \gamma_{1}=0.01
$$

\section{Model prediction}

Substitution of the dispersion closure (3.17) into (2.10) and (2.11) results in

$$
\begin{gathered}
\frac{\partial Q_{m}}{\partial t}+\frac{\partial M_{m}}{\partial z}=0 \\
\frac{\partial M_{m}}{\partial t}+\gamma_{0} \frac{\partial}{\partial z}\left(\frac{M_{m}^{2}}{Q_{m}}\right)=-2 \alpha_{0} \gamma_{0} \frac{M_{m}^{5 / 2}}{Q_{m}^{2}}+6 \gamma_{1} \frac{\partial}{\partial z}\left[\frac{M_{m}^{2}}{Q_{m}}+\frac{Q_{m}^{2}}{4 \alpha_{0} M_{m}^{1 / 2}} \frac{\partial}{\partial z}\left(\frac{M_{m}^{2}}{Q_{m}^{2}}\right)\right],
\end{gathered}
$$

where we have used the steady-state relationship $\delta_{m}=-2 \alpha_{0} \gamma_{0}$. Notably, (4.1) and (4.2) admit well-established power-law solutions describing a steady state. The addition of the final term in (4.2) affects neither the exponent of the steady-state solutions nor the predicted spreading rate of the jet. The system (4.1) and (4.2) can be readily solved numerically using a central differencing scheme. 
In this section we compare the predictions of our model (4.1) and (4.2) and the modified model of Scase \& Hewitt (2012), described by (2.15) and (2.16), which will hereafter be referred to as the Gaussian jet model (GJM) and the top-hat jet model (TJM), respectively, to the DNS results obtained in Part 1. To solve the system (4.1) and (4.2) for GJM we discretise spatial derivatives by taking second-order central differences and advance the solution in time using a fourth-order Runge-Kutta method. At the base of the domain we prescribe unsteady source conditions $Q_{m}(0, t)=Q_{0}(t)$ and $M_{m}(0, t)=M_{0}(t)$. In particular, we impose a rapid smooth increase or decrease in the source momentum flux according to

$$
M_{0}(t)=M_{0}^{B}+\frac{1}{2}\left(M_{0}^{A}-M_{0}^{B}\right)\left[1+\operatorname{erf}\left(\frac{t-t_{v}}{t_{*}}\right)\right],
$$

where $M_{0}^{B}$ and $M_{0}^{A}$ are the source momentum flux before and after the sudden change, respectively, and we ensure that $t_{*}$ is approximately two orders of magnitude smaller than the timescale relevant to the source. During this change we hold the source radius, $r_{0}=Q_{0} / M_{0}^{1 / 2}$, constant and therefore adjust $Q_{0}$ accordingly. The time $t_{v}$ is the temporal location of an asymptotic virtual source and was determined from the DNS data in Part 1. For relatively large values of $z$ we assume that any dependent variable $X_{m}$ behaves according to the steady state:

$$
\lim _{z \rightarrow \infty} \frac{\partial X_{m}}{\partial z}=\frac{n}{z-z_{v}} X_{m}
$$

where $X_{m} \sim z^{n}$ in the steady state and $z_{v}$ is the location of a virtual origin. In practice, (4.4) is approximated numerically, and imposed on all dependent variables at a value of $z$ sufficiently large to ensure that the condition does not interfere with the unsteady solution. The equations are solved over the time domain $\left[t_{v}-\delta t, t_{\infty}\right]$, where $\delta t=O\left(t^{*}\right)$, which ensures that the transition (4.3) is smooth and approximately symmetric, and $t_{\infty}$ is a time that is approximately equal to the duration of the DNS to which we compare. The DNS data were obtained from an ensemble average of individual, statistically unsteady, simulations. Further details about the cases presented can be found in Part 1 .

To obtain numerical solutions to the adjusted top-hat model proposed by Scase \& Hewitt (2012), TJM, we set $\beta_{1}=3 \gamma_{1}$, so that the diffusive flux in (2.21) is equal to the dispersive flux in our model. For TJM a central differencing scheme proved impractical, and the flux-limiting scheme described by Kurganov \& Tadmor (2000) was employed instead. The results presented in Scase \& Hewitt (2012) were also obtained via the use of a flux-limiting scheme.

The numerical approximation to both GJM and TJM was performed using a grid of 2000 points over the domain $z / r_{0} \in[0,89]$. In addition, we checked convergence by halving the total number of points that were used. In figures 1 and 2 the full- and halfresolution approximations are shown with lines and symbols, respectively. We note that the use of 2000 points, which makes the calculation computationally expensive, was necessitated by the difficulty of achieving convergence with TJM. The timescale $\tau_{0}$ for the unsteady simulations was defined according to $\tau_{0} \equiv r_{0}^{2} / M_{m}^{* 1 / 2}$, where $M_{m}^{* 1 / 2}$ is a momentum flux that characterises the motion of the front (see Part 1 or $\S 5$ in this paper). The DNS data shown in figures 1 and 2 were obtained from integrals of ensemble-averaged quantities.

Figure 1 indicates that the unsteady model developed here, GJM, provides an excellent description of both the propagation speed and the spreading rate of both 

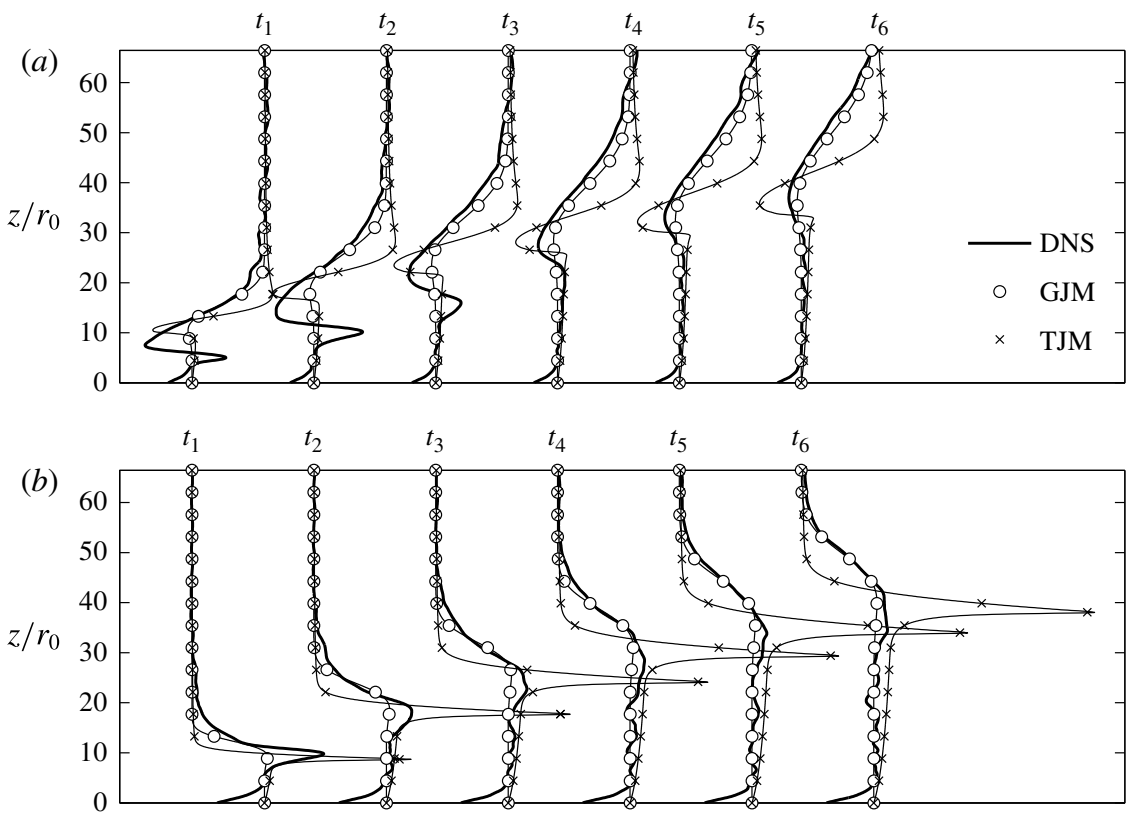

FIgURE 1. Mean momentum flux $M_{m}$ following (a) a step-down and (b) a step-up in the momentum flux at the source. Direct numerical simulation compared with theoretical prediction at times approximately given by $t_{n}=18 \tau_{0} n$.

types of front in $M_{m}$. In contrast, TJM significantly under- and overpredicts the value of $M_{m}$ upstream of the front in the step-down case (figure 1a) and the step-up case (figure $1 b$ ), respectively. In particular, the spike in $M_{m}$ predicted by TJM is extremely difficult to approximate numerically and appears to be non-physical. At the level of the front TJM overpredicts and underpredicts $M_{m}$, in the step-down and the step-up case, respectively, and displays a poor agreement with the slope that is observed in the DNS data. Furthermore, the inconsistency of TJM with the steady-state solution is evidenced in the increase in $M_{m}$ that occurs upstream of the front. Notable from the DNS results are the local maxima in $M_{m}$ in the near-field region, $z / r_{0}<20$, which GJM does not reproduce. Indeed, close to the source the jet has a top-hat profile, which slowly decays into an approximately Gaussian profile as the flow develops (Part 1). Accordingly, unsteady top-hat models can reproduce certain features of the flow in the near field but do not accurately capture its far-field behaviour.

From figure 1, one may be inclined to suppose that a larger value of the diffusion coefficient $\beta_{1}$ in TJM would give an improved agreement with the DNS data. In fact, increasing $\beta_{1}$ has an adverse effect on the predictive capabilities of TJM because it increases the strength of the spurious source of momentum and energy that was identified in (2.17). A substantial increase in $\beta_{1}$ results in an overestimation of the total mean flow energy in the domain and, consequently, an overestimation of the propagation speed of the front. Additionally, with a significant increase in its value, $\beta_{1}$ would exceed the bounds that are implied by the values of eddy viscosity on which it is based (Scase \& Hewitt 2012).

The local radius of the jet is a useful quantity to examine because, representing a balance between $Q_{m}$ and $M_{m}^{1 / 2}$, its behaviour is not easy to anticipate a priori. The radius is also significant as the square root of the 'conserved' quantity in the mass 

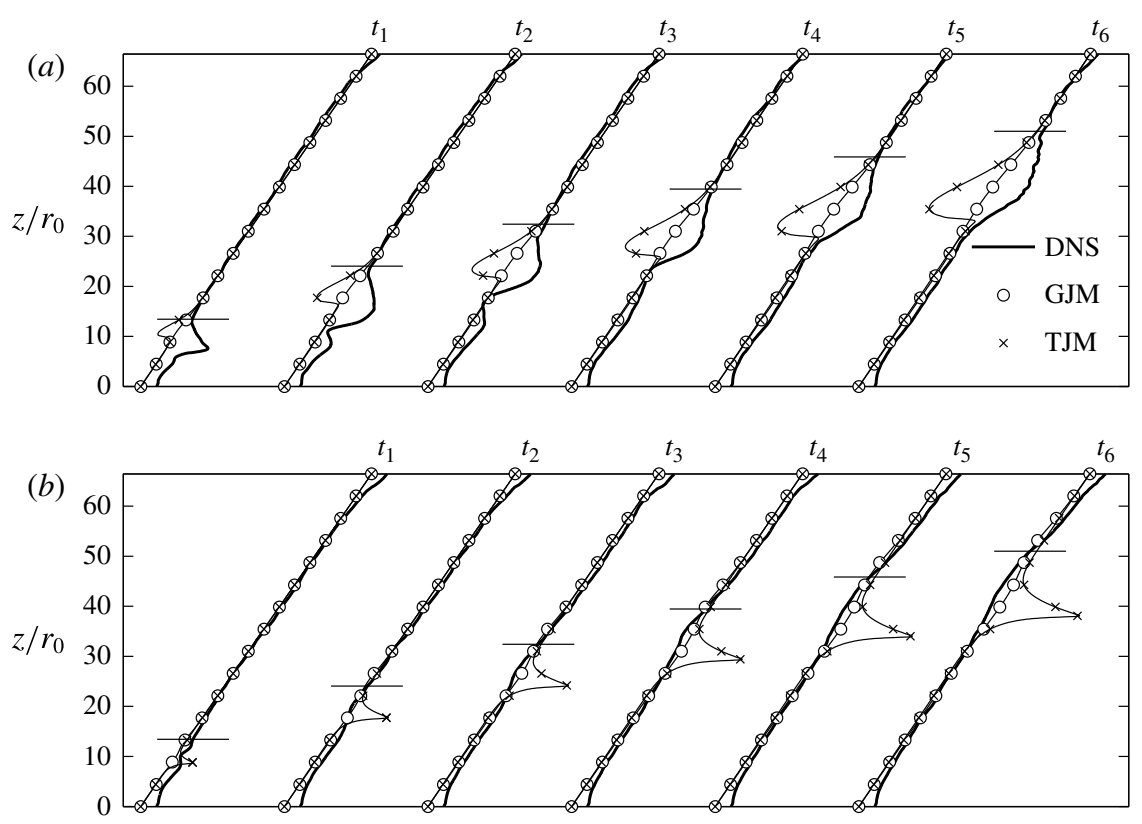

FIGURE 2. Jet radius $r_{m} \equiv Q_{m} / M_{m}^{1 / 2}$ following (a) a step-down and (b) a step-up in the momentum flux at the source. Direct numerical simulation compared with theoretical prediction at times approximately given by $t_{n}=18 \tau_{0} n$. The location of the front is indicated with a horizontal line.

conservation equation (2.15). Perhaps surprisingly, figure 2(b) shows that the radius of the jet is approximately unaffected by the local increase in momentum flux, and is well-predicted by GJM. In contrast, TJM predicts a significant local increase in the radius in figure $2(b)$. In $\S 6.2$ we show that this property of TJM is due to the fact that it assumes a top-hat, rather than Gaussian, distribution of longitudinal velocity. In figure 2(a), which displays the step-down case, the DNS data reveal that the jet radius $r_{m}$ increases locally behind the front, which is not captured by GJM and is in direct contradiction with the reduction in radius predicted by TJM. However, it is helpful to note that the local increase in area observed in the DNS occurs upstream of the leading edge of the front and corresponds to the local minimum in $M_{m}$ in figure $1(a)$. By implication, since $r_{m} \equiv Q_{m} / M_{m}^{1 / 2}, Q_{m}$ appears to be relatively insensitive to the changes that occur in this region. Furthermore, relative to the steady-state jet radius $2 \alpha_{0} z$, the local increase in $r_{m}$ that is evident in figure 2(a) diminishes in amplitude as $z$ increases. The physics associated with the step-down case appear to be quite different to the step-up case and accurate prediction of the region upstream of the leading front, particularly in the near field, represents a challenge for a single, two-variable integral model. It should also be noted that the local increase in $r_{m}$ seen in figure 2(a) corresponds to an increase in the threshold radius $r_{d}$ at the same level.

In making an assessment of the models' ability to predict the radius of the unsteady jets it is necessary to bear in mind that $r_{m} \equiv Q_{m} / M_{m}^{1 / 2}$ is not the same as the 'effective top-hat' radius employed by Scase, Caulfield \& Dalziel (2008) and Scase et al. (2009). The 'effective top-hat' radius, defined in Scase et al. (2008), is based on the total concentration of a passive tracer at a given height. Indeed, the use of a passive tracer is a valuable aid in experiments that do not permit direct access to the velocity field. 
However, the 'effective top-hat' radius will not, in general, coincide with $r_{m}$, which is the only length scale that can be formed from $Q_{m}$ and $M_{m}$. With full access to the velocity field from the DNS data, we focus directly on a comparison of the models' prediction of $Q_{m}$ and $M_{m}$, or any variable, such as $r_{m}$, that can be expressed in terms of $Q_{m}$ and $M_{m}$. Consequently, we note that the behaviour of $r_{m}$ shown in figure 2 is not inconsistent with the narrowing of an unsteady plume observed by Scase et al. (2008), since the latter was inferred from the 'effective top-hat' radius.

Collectively, figures 1 and 2 suggest that a top-hat description of unsteady jets does not reproduce observations satisfactorily and that the dispersion model of GJM is physically more realistic. We have also pointed out that top-hat models, such as TJM, are difficult to solve numerically, in spite of the fact that they are rendered well-posed with the inclusion of a longitudinal mixing term. The reason for this difficulty is that they do not contain type I dispersion. In other words, they invoke the non-physical assumption that velocity profiles are uniform. In $\S 6$ we explain the consequences of this issue in more detail, showing that if the velocity profiles are assumed to be non-uniform $\left(\gamma_{m}>1\right)$ perturbations applied at the source of the jet have an algebraic downstream growth, or even decay, in place of the exponential growth associated with top-hat profiles. Furthermore, we show that when $\gamma_{m}<4 / 3$, the area upstream of a sudden increase in the momentum flux increases, allowing the jet to store momentum and energy locally.

\section{A straight-sided approximation}

For problems involving a step change in $M_{m}$ at a source of constant area, $A_{0}$, it is natural to ask in what way the area in the vicinity of the front $\left(z=z^{*}(t)\right)$ departs from its steady-state form. When $z / z^{*} \ll 1$ and $z / z^{*} \gg 1$, we expect the area to remain unchanged, so that $A_{m}=Q_{m}^{2} / M_{m}=4 \alpha_{0}^{2} z^{2}$. In their unsteady plume model, Vul'fson \& Borodin (2001) go further and assume that the area is independent of time for all $z$, and therefore assumes its steady-state form. In this section we will show that for jets, such a 'straight-sided' assumption can be justified theoretically and has several fundamental implications for the governing system of equations. We will assume that the source area $A_{0}$ remains fixed. In $\S 5.1$ we will neglect type II dispersion and therefore assume that $\gamma_{m}$ is a constant equal to $\gamma_{0}$. In $\S 5.2$ we extend the analysis to include type II dispersion and obtain a simple advection-dispersion equation that serves as a good approximation to the full model (4.1) and (4.2).

\subsection{A straight-sided equation for momentum and energy (type I dispersion only)}

The unsteady equation for area (2.9), neglecting type II dispersion (i.e. $\gamma_{m}=\gamma_{0}$ ) and using (2.12), can be expressed as

$$
\frac{\partial A_{m}}{\partial t}=-\gamma_{0} \frac{\partial Q_{m}}{\partial z}+2 \alpha_{0} \gamma_{0} M_{m}^{1 / 2}+\frac{2 Q_{m}}{M_{m}}\left(\gamma_{0}-1\right) \frac{\partial M_{m}}{\partial z} .
$$

Substitution of the straight-sided solution $Q_{m}=2 \alpha_{0} M_{m}^{1 / 2} z$ into (5.1) reveals that

$$
\frac{\partial A_{m}}{\partial t}=\frac{4 \alpha_{0} z}{M_{m}^{1 / 2}}\left(\frac{3}{4} \gamma_{0}-1\right) \frac{\partial M_{m}}{\partial z} .
$$

Equation (5.2) expresses the fact that when $\gamma_{0}=4 / 3$ the jet will remain straightsided for all time. Further, (5.2) suggests that when $\gamma_{0}<4 / 3$ or $\gamma_{0}>4 / 3$ the area 
will increase or decrease, respectively, following a step increase in $M_{m}$. Indeed, the unsteady similarity solutions obtained by Scase et al. (2006) can be rederived for arbitrary $\gamma_{0}$ (see appendix A) and reveal that when $\gamma_{0}=4 / 3$, the spreading angle of the jet is identical to the steady-state case: $r_{m}=2 \alpha_{0} z$. In addition, the similarity solutions show that when $\gamma_{0}<4 / 3$ or $\gamma_{0}>4 / 3$ the spreading angle of the jet is less than or greater than the steady-state spreading angle, respectively. When $\gamma_{0}=1$, one recovers the similarity solution for top-hat profiles obtained by Scase et al. (2006) and the behaviour $r_{m}=2 \alpha_{0} z / 3$. Contrary to the top-hat plume theory in which there is no means for faster fluid to overtake slower fluid (see e.g. Scase et al. 2009), we find that specification of a realistic, albeit constant, value of $\gamma_{0}$ facilitates such overtaking in the form of type I dispersion.

In the light of (5.2), the Gaussian velocity profile can be usefully understood as providing momentum and energy fluxes whose ratio is such that the steady-state area of the jet can be preserved. To visualise this one can consider a control volume located at the front of a starting jet. Energy, $M_{m}$, is transported into the control volume with dimensionless flux $\gamma_{0}$, and it is removed from the control volume owing to turbulence production at a dimensionless rate $\delta_{m}$. If $\gamma_{0}<4 / 3$ then energy is added to the control volume at a reduced rate, which results in a relative surplus of momentum, $Q_{m}$, and hence the accumulation of area $Q_{m}^{2} / M_{m}$. Conversely, $\gamma_{0}>4 / 3$ results in the reduction or dispersion of area.

The use of the straight-sided approximation is highly desirable from a modelling perspective, as it provides an algebraic coupling between $Q_{m}$ and $M_{m}$ and therefore reduces the dimension of the system by one. For straight-sided jets, using the relations $Q_{m}=2 \alpha_{0} M_{m}^{1 / 2} z$ and $\delta_{m}=-8 \alpha_{0} / 3$, which both follow from $\gamma_{0}=4 / 3$, the momentum equation and mean energy equations (2.10) and (2.11) become

$$
\begin{gathered}
\frac{\partial Q_{m}}{\partial t}+\frac{\partial}{\partial z}\left(\frac{Q_{m}^{2}}{4 \alpha_{0}^{2} z^{2}}\right)=0 \\
\frac{\partial M_{m}}{\partial t}+\frac{2}{3} \frac{\partial}{\partial z}\left(\frac{M_{m}^{3 / 2}}{\alpha_{0} z}\right)=-\frac{2}{3} \frac{M_{m}^{3 / 2}}{\alpha_{0} z^{2}} .
\end{gathered}
$$

Both (5.3) and (5.4) admit discontinuities at $z=z^{*}(t)$ that propagate according to

$$
\frac{\mathrm{d} z^{*}}{\mathrm{~d} t}=\frac{Q_{m}^{*}}{2 \alpha_{0}^{2} z^{* 2}}=\frac{M_{m}^{* 1 / 2}}{\alpha_{0} z^{*}} .
$$

The nonlinearity of (5.3) and (5.4) ensures that a positive step change $\left(\partial_{z} M_{m}<0\right)$ in $M_{m}$ gives rise to a shock, whose propagation velocity must be determined from a Rankine-Hugoniot condition (see e.g. Toro 1997). In order to determine a unique propagation velocity one must choose whether to impose momentum conservation or energy conservation over the shock. Likening the progression of a positive step change in a jet's momentum flux to the evolution of a tidal bore, we impose momentum conservation (5.3) over the discontinuity. Substituting $Q_{m}=2 \alpha_{0} M_{m}^{1 / 2} z$ in (5.3), we find

$$
M_{m}^{* 1 / 2}=\frac{1}{2}\left(\frac{M_{m}^{A}-M_{m}^{B}}{M_{m}^{A 1 / 2}-M_{m}^{B 1 / 2}}\right) .
$$

Here, we use the superscripts $B$ and $A$ to denote the value of a variable or function before/ahead of and after/behind a front, respectively. The characteristic momentum flux (5.6) provided the motivation for the choice of scaling in figure 10 of Part 1. 
It is also of interest that for a starting jet, for which $M_{m}^{B}=0$, (5.6) is consistent with the result obtained by Ruban \& Vonatsos (2008). There, the Bernoulli equation was used along the axis of a planar jet to deduce that the propagation velocity is equal to half the maximum velocity in the jet immediately behind the shock. We note, however, that in other respects their laminar boundary layer solution is very different to the radially integrated turbulent flow considered here. In the present work, if $M_{m}^{B}=0$ in (5.6) then $\mathrm{d} z^{*} / \mathrm{d} t=w_{m}^{A}$, where $w_{m}^{A}$ is the top-hat velocity immediately behind the shock. Noting that the top-hat velocity $w_{m}^{A}$ is equal to half the maximum velocity of a Gaussian profile confirms the consistency between (5.6) and the results of Ruban \& Vonatsos (2008). In turn, these deductions each agree closely with the observations reported by Turner (1962) for a starting plume.

In (5.4) the inclusion of type I dispersion alone, and not the mixing provided by type II dispersion, has produced a simple first approximation of an unsteady jet. In spite of the absence of longitudinal mixing, (5.4) does not admit the non-physical growth of short-wave modes that were found in the predictions of several unsteady plume models (Scase \& Hewitt 2012). Indeed, we show in $\S 6.1$ that the evolution of short-wave modes is determined by type I dispersion, and that their growth can therefore be prevented by adopting a physically realistic assumption regarding the jet's velocity profile.

Although it is clear that the mixing in jets prevents the formation of discontinuities, like other hyperbolic systems, the value of (5.3) or (5.4) is that it provides a description of their limiting behaviour, without the further complications that are introduced when additional mixing terms are present.

\subsection{A straight-sided equation for energy (type I and type II dispersion, approximate momentum conservation)}

In (5.3) and (5.4) we established a description of a straight-sided unsteady jet that satisfies transport equations for both momentum and mean energy. In general, if the straight-sided relation $Q_{m}=2 \alpha_{0} M_{m}^{1 / 2} z$ is imposed strictly then one cannot expect to satisfy equations for momentum and mean energy simultaneously unless $\gamma_{m}=4 / 3$. Indeed, the solution for the two dependent variables of the problem is defined by two governing equations. The Gaussian profile, for which $\gamma_{m}=4 / 3$, is the distinguished case for which the three equations (momentum, mean energy and straight-sidedness) are consistent.

As an approximate treatment it is useful to relax the momentum equation and consider a solution that satisfies both straight-sidedness and the mean energy equation exactly. The extent to which the momentum equation will be violated in this approximation depends on the value of $\gamma_{0}$ and $\gamma_{1}$. In particular, if we let $\gamma_{0}=4 / 3$, then the level of approximation in the momentum equation depends on the amount of longitudinal mixing, and therefore on $\gamma_{1}$, whose value is small in comparison to 4/3. Approximating the momentum balance in this way allows us to obtain a straight-sided version of the full unsteady jet model developed in $\S 3$, which reduces to a simple advection-dispersion equation. As such, it can be readily employed to aid a first estimation of a dispersion coefficient based on experimental or numerical observations.

With the straight-sided solution $Q_{m}=2 \alpha_{0} M_{m}^{1 / 2} z$, our model for energy transport (4.2) simplifies to a nonlinear advection-dispersion equation:

$$
\frac{\partial M_{m}}{\partial t}+\frac{3 \gamma_{0}}{4 \alpha_{0}} \frac{M_{m}^{1 / 2}}{z} \frac{\partial M_{m}}{\partial z}=\frac{3 \gamma_{1}}{2 \alpha_{0}} \frac{\partial}{\partial z}\left(M_{m}^{1 / 2} \frac{\partial M_{m}}{\partial z}\right) .
$$


To understand the leading-order processes described by (5.7) we consider dimensionless perturbations from $M_{m}=M_{m}^{*}$ :

$$
M_{m}=M_{m}^{*}\left(1+\epsilon \mathscr{M}_{1}+\epsilon^{2} \mathscr{M}_{2}+\cdots\right) .
$$

Here, we have employed the characteristic momentum flux $M_{m}^{*}$, rather than $M_{m}^{B}$ for example, because it is $M_{m}^{*}$ that determines the propagation velocity of the front. In doing so, we find that the linearised system of equations obtained below can provide a good approximation to the fully nonlinear problem, in which the step-change forcing is relatively large. Substituting this expansion into (5.7) and retaining terms to $O(\epsilon)$ yields

$$
\frac{\partial \mathscr{M}_{1}}{\partial t}+\frac{3 \gamma_{0}}{4 \alpha_{0}} \frac{M_{m}^{* 1 / 2}}{z} \frac{\partial \mathscr{M}_{1}}{\partial z}=\frac{3 \gamma_{1}}{2 \alpha_{0}} M_{m}^{* 1 / 2} \frac{\partial^{2} \mathscr{M}_{1}}{\partial z^{2}},
$$

which is a linear advection-diffusion equation for a spatially varying velocity field, and has exactly the same form as the equation that was used in Part 1 to estimate the mixing coefficient $D_{e}$. Indeed, comparison of (5.9) above with (5.9) in Part 1 shows that $D_{e} / M_{m}^{* 1 / 2}=3 \gamma_{1} /\left(2 \alpha_{0}\right)$. Assuming similarity of the process in terms of the variable $\lambda$ :

$$
\lambda \equiv \frac{z^{2} \alpha_{0}}{t M_{m}^{* 1 / 2}},
$$

where the dimensionless front velocity $\lambda\left(z^{*}\right)=\lambda^{*}=2$, (5.9) can be expressed as

$$
\frac{\mathrm{d}^{2} \mathscr{M}_{1}}{\mathrm{~d} \lambda^{2}}=\frac{1}{2 \lambda}\left[P e\left(1-\frac{\lambda}{\lambda^{*}}\right)-1\right] \frac{\mathrm{d} \mathscr{M}_{1}}{\mathrm{~d} \lambda},
$$

where the dispersive Péclet number

$$
P e \equiv \frac{\gamma_{0}}{2 \gamma_{1}}
$$

is consistent with the definition provided in appendix B of Part 1. Indeed, in Part 1 the theoretical justification for using the linear advection-dispersion equation was not provided, yet it was found to give a reasonable agreement with the data. As described in this section, it is the tendency of the jet to remain straight-sided that results in the surprising effectiveness of (5.9). The Péclet number defined in (5.12) has a meaning that is equivalent to the ratio of advection and dispersion parameters $\left(K_{a} / K_{d}\right)$ used in the recent study of a quasi-two-dimensional jet by Landel et al. (2012).

In figure 3 we display self-similar, far-field predictions from the general nonlinear model GJM, the straight-sided model GJM-S (5.7), and the linear straight-sided model GJM-SL (5.9), in addition to the collapsed DNS data. In GJM-S and GJM-SL we set $\gamma_{0}=4 / 3$, which is consistent with the value of $\gamma_{m}$ required for straight-sidedness. We do not include predictions for TJM, as it proved infeasible to provide the number of points that were required for a far-field solution in that case. Notably, having been obtained from the constraint $r_{m}=2 \alpha_{0} z$, both the linear and nonlinear straight-sided solutions are monotonic, and are therefore not able to reproduce the extrema evident in both the DNS results and the full model. As one might expect, in the step-up (stepdown) case the nonlinear model GJM-S predicts a steeper (less steep) front than the linear approximation GJM-SL. The linear, GJM-SL, and nonlinear, GJM-S, straightsided predictions exhibit a good agreement, which demonstrates the effectiveness of the former when $M_{m}^{*}$ is chosen correctly, i.e. as the solution of the idealised nonlinear 

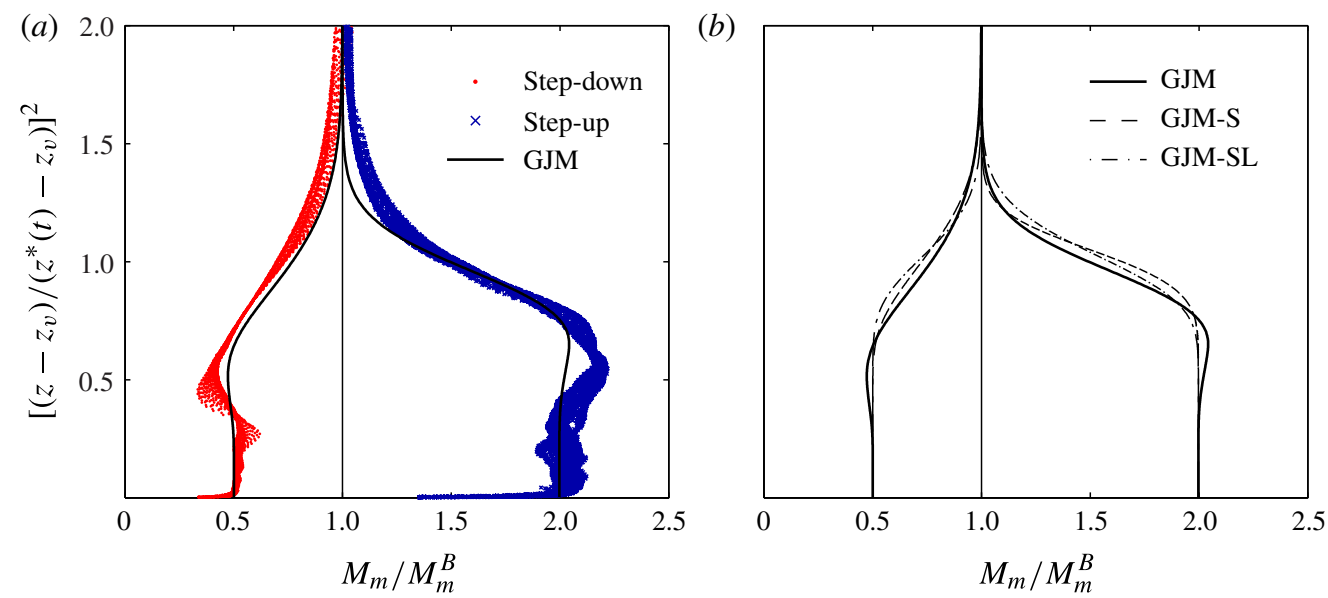

FIGURE 3. (Colour online) (a) Self-similarity of the dimensionless momentum flux $M_{m} / M_{m}^{B}$ from DNS results compared to predictions using the full model GJM (4.1) and (4.2). (b) Predictions obtained using the nonlinear, straight-sided model GJM-S (5.7), and linear straight-sided model GJM-SL (5.9) are compared to GJM. The constant $z_{v}$ is the value of $z$ at an asymptotic virtual source. In each plot, the left-hand side refers to the step-down case, and the right-hand side to the step-up case.

shock problem. It is also evident in figure 3 that the full model GJM is not able to exactly reproduce the values $M_{m} / M_{m}^{B}>1$ at relatively large values of $z / z^{*}$ in the stepup case. An attempt to correct this by increasing $\gamma_{1}$ would mean that $\gamma_{1}$ no longer faithfully represents those features of the flow on which its definition in $\S 3.2$ was originally based.

\section{The hyperbolic jet}

In this section we move back to the original system (2.10) and (2.11) and investigate several properties of the hyperbolic system that one obtains by assuming that the parameter $\gamma_{m}$ remains constant. With this assumption we therefore neglect type II dispersion and focus exclusively on type I dispersion. The justification for this restriction is that type I dispersion is of leading order and has several interesting properties that are readily exposed in a simplified setting. In addition, the use of a constant value of $\gamma_{m}$ is equivalent to the approach taken in previous unsteady jet and plume models. In contrast to the previous sections, whose focus was on modelling the various transport processes, the purpose of this section is to investigate the parameter space $\gamma_{m}$. Here we make no assumption regarding straight-sidedness and ascribe to the jet an arbitrary radial profile of longitudinal velocity.

In particular, we will demonstrate that type I dispersion determines the growth rate of perturbations in the jet, which pertains to the analysis performed by Scase \& Hewitt (2012) for plumes. In addition, we will show that type I dispersion is responsible for the behaviour of $r_{m}$ and $w_{m}$ behind the leading front of the hyperbolic problem that one obtains by assuming that $\gamma_{m}$ depends on neither $z$ nor $t$. Although we have emphasised that in real jets $\gamma_{m} \sim 4 / 3$, understanding the way in which $\gamma_{m}<4 / 3$ and $\gamma_{m}>4 / 3$ influences the behaviour of an unsteady jet is valuable for several reasons. First, it explains why models that assume a top-hat distribution of velocities admit the 
unbounded growth of disturbances. Secondly, it provides a tentative indication as to why the velocities in a jet might naturally converge towards a Gaussian distribution.

\subsection{The growth rate of source perturbations}

In order to determine the cause of the difficulties associated with several unsteady plume models, Scase \& Hewitt (2012) analysed their response to infinitesimal perturbations of the source conditions. Here we repeat the analysis for unsteady jets, focusing on a single harmonic of frequency $\sigma$. It is convenient to work in terms of the dimensionless variables

$$
\zeta \equiv \frac{\alpha_{0} z^{2} \sigma}{M_{m 0}^{1 / 2}}, \quad \tau \equiv \sigma t
$$

where $M_{m 0}$ is a constant steady-state momentum flux. We will assume that the dimensionless energy flux $\gamma_{m}$ is constant. In terms of the dimensionless variables $(6.1 a, b)$, linearised equations enforcing the conservation of momentum and energy, in terms of the dimensionless volume flux $\mathscr{Q}_{1}$ and momentum flux $\mathscr{M}_{1}$, can be expressed as

$$
\left(\frac{\partial}{\partial \tau}+\left[\begin{array}{cc}
0 & 1 \\
-\gamma_{m} & 2 \gamma_{m}
\end{array}\right] \frac{\partial}{\partial \zeta}-\left[\begin{array}{cc}
0 & 0 \\
\frac{\gamma_{m}}{2 \zeta} & -\frac{\gamma_{m}}{4 \zeta}
\end{array}\right]\right)\left(\begin{array}{l}
\mathscr{Q}_{1} \\
\mathscr{M}_{1}
\end{array}\right)=\left(\begin{array}{l}
0 \\
0
\end{array}\right)
$$

We decompose the problem into Fourier modes and, focusing on the harmonic of frequency $\sigma$, assume a solution of the form

$$
\left(\begin{array}{c}
\mathscr{Q}_{1} \\
\mathscr{M}_{1}
\end{array}\right)=\left(\begin{array}{c}
\hat{\mathscr{Q}}_{1}(\zeta) \\
\hat{\mathscr{M}}_{1}(\zeta)
\end{array}\right) \exp (\mathrm{i} \tau)
$$

It follows from (6.2) that

$$
\frac{\mathrm{d}^{2} \hat{\mathscr{M}}_{1}}{\mathrm{~d} \zeta^{2}}+\left(2 \mathrm{i}+\frac{1}{2 \zeta}\right) \frac{\mathrm{d} \hat{\mathscr{M}}_{1}}{\mathrm{~d} \zeta}+\left(\frac{\mathrm{i}}{4 \zeta}-\frac{1}{\gamma_{m}}\right) \hat{\mathscr{M}}_{1}=0
$$

which is a confluent hypergeometric equation, whose general solution is

$$
\hat{\mathscr{M}}_{1}(\zeta)=\exp [-\mathrm{i} \zeta(1+\phi)] \sqrt{\zeta}\left[c_{1} \mathrm{M}\left(\frac{6 \phi+1}{8 \phi}, \frac{3}{2}, 2 \zeta \phi \mathrm{i}\right)+c_{2} \mathrm{U}\left(\frac{6 \phi+1}{8 \phi}, \frac{3}{2}, 2 \zeta \phi \mathrm{i}\right)\right]
$$

where $\mathrm{M}$ and $\mathrm{U}$ are independent solutions of Kummer's equation (Abramowitz \& Stegun 1972, p. 504), $c_{1}$ and $c_{2}$ are arbitrary constants, and

$$
\phi \equiv \sqrt{1-\frac{1}{\gamma_{m}}}
$$

The limiting form of (6.5) for large $z$ is given by Abramowitz \& Stegun (1972, p. 508):

$$
\hat{\mathscr{M}}_{1}(\zeta) \sim c_{1} \exp [\mathrm{i} \zeta(\phi-1)] \frac{\Gamma(3 / 2)}{\Gamma\left(\frac{6 \phi+1}{8 \phi}\right)}(2 \phi \mathrm{i})^{a(\phi)-1 / 2} \zeta^{a(\phi)}
$$




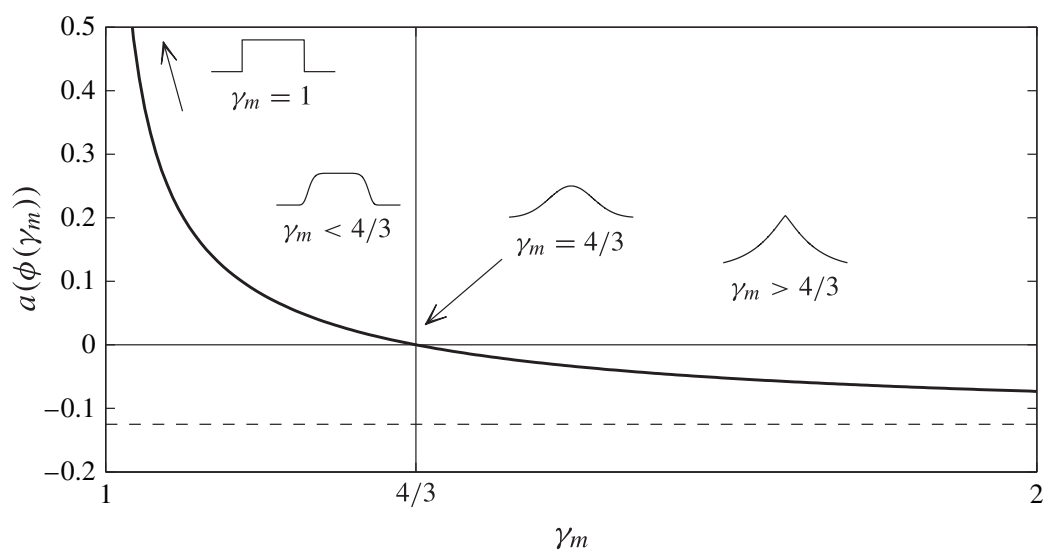

FIGURE 4. Dependence of power-law growth of source perturbations applied to a jet on the dimensionless energy flux $\gamma_{m}$. The dashed line indicates the value $-1 / 8$ of $a(\phi)$ as $\phi \rightarrow \infty$.

The asymptotic solution (6.7) reveals the growth rate of the perturbations in $\zeta$, with exponent

$$
a(\phi)=\frac{1}{8 \phi}-\frac{1}{4},
$$

which is displayed in figure 4 . When $\gamma_{m}>4 / 3$ the exponent is less than one and the perturbations decay. However, when $\gamma_{m}<4 / 3$ the perturbations grow without bound. The Gaussian profile, or straight-sided jet, for which $\gamma_{m}=4 / 3$, is the special case for which the amplitude of the perturbations remains constant. It is tempting to speculate that it is in order to yield constant-amplitude perturbations that the flow chooses to be Gaussian in form. In the limit $\gamma_{m} \rightarrow 1$ the perturbations grow exponentially, and the limiting form of the solution is

$$
\hat{\mathscr{M}}_{1}(\zeta) \sim c_{3} \exp \left[\left(\frac{1}{2}+\frac{\mathrm{i}}{2}\right) \sqrt{2 \zeta}-\mathrm{i} \zeta\right],
$$

whilst in the straight-sided limit $\gamma_{m} \rightarrow 4 / 3$ the solution degenerates into a single harmonic of constant amplitude:

$$
\hat{\mathscr{M}}_{1}(\zeta) \sim c_{4} \exp \left(-\mathrm{i} \frac{\zeta}{2}\right),
$$

for constants $c_{3}$ and $c_{4}$.

\subsection{The response of the jet area}

Under the assumption that $\gamma_{m}$ remains constant, characteristic curves of the system (2.10) and (2.11) are paths along which the total derivatives of several quasi-invariant quantities are decoupled. Using the eigenvectors of the system the invariants are defined in terms of the total derivatives (Part 1):

$$
\mathrm{d} Y_{m}^{n}=-\frac{\gamma_{m}}{\lambda_{n}^{*}} \frac{M_{m}}{Q_{m}} Q_{m}^{-\gamma_{m} / \lambda_{n}^{*}} \mathrm{~d} Q_{m}+Q_{m}^{-\gamma_{m} / \lambda_{n}^{*}} \mathrm{~d} M_{m},
$$


where the integrating factor $Q_{m}^{-\gamma_{m} / \lambda_{n}^{*}}$ has been introduced. Here $\lambda_{n}^{*}$ is an eigenvalue of the original system of equations (see Part 1, equation (5.4)):

$$
\lambda_{n}^{*}=\gamma_{m}\left[1+(-1)^{n}\left(1-\frac{1}{\gamma_{m}}\right)^{1 / 2}\right], \quad n=1,2,
$$

where $n=1$ and $n=2$ correspond to the slower and faster of the two characteristic curves, respectively. Consequently, the quasi-invariants are

$$
Y_{m}^{n}=\frac{M_{m}}{Q_{m}^{\gamma_{m} / \lambda_{n}^{*}}}
$$

If we consider a case in which the turbulence production is zero, then the original governing equations (2.10) and (2.11) become a homogeneous system and $\mathrm{d} Y_{m}^{n} / \mathrm{d} t=0$ along characteristic curves. Steady-state solutions to this idealised case correspond to a jet that does not entrain and therefore has a constant volume flux and a constant momentum flux. If a discontinuity is imposed at $(z, t)=(0,0)$ then $Q_{m}$ and $M_{m}$ will be constant in a region $S$, bounded by fast and slow characteristic curves that emanate from $(0,0)$. Therefore, in the homogeneous case, the value of the invariants, and hence the values $Q_{m}^{S}$ and $M_{m}^{S}$, of $Q_{m}$ and $M_{m}$ in $S$, respectively, can be determined by tracing fast and slow characteristic curves to points outside $S$ at which the solution is known:

$$
\frac{M_{m}^{S}}{\left(Q_{m}^{S}\right)^{\gamma_{m} / \lambda_{1}^{*}}}=\frac{M_{m}^{B}}{\left(Q_{m}^{B}\right)^{\gamma_{m} / \lambda_{1}^{*}}}, \quad \frac{M_{m}^{S}}{\left(Q_{m}^{S}\right)^{\gamma_{m} / \lambda_{2}^{*}}}=\frac{M_{m}^{A}}{\left(Q_{m}^{A}\right)^{\gamma_{m} / \lambda_{2}^{*}}} .
$$

The solution of these equations is

$$
M_{m}^{S}=X_{m}^{1 /(2 \phi)} M_{m}^{A}, \quad Q_{m}^{S}=X_{m}^{1 /(2 \phi)+1 / 2} Q_{m}^{A},
$$

where $\phi$ was defined in (6.6) and

$$
X_{m} \equiv\left(\frac{M_{m}^{A}}{M_{m}^{B}}\right)^{1-\phi}\left(\frac{Q_{m}^{B}}{Q_{m}^{A}}\right) .
$$

Alternatively, $(6.15 a, b)$ can be manipulated to give the velocity $w_{m}^{S}$ and radius $r_{m}^{S}$ in the region $S$,

$$
w_{m}^{S}=X_{m}^{-1 / 2} w_{m}^{A}, \quad r_{m}^{S}=X_{m}^{1 /(4 \phi)+1 / 2} r_{m}^{A},
$$

where

$$
X_{m} \equiv\left(\frac{w_{m}^{A}}{w_{m}^{B}}\right)^{1-2 \phi}\left(\frac{r_{m}^{B}}{r_{m}^{A}}\right)^{2 \phi} .
$$

If it is assumed that the area is held constant at the source, then

$$
w_{m}^{S}=\left(\frac{w_{m}^{A}}{w_{m}^{B}}\right)^{\phi-1 / 2} w_{m}^{A}, \quad r_{m}^{S}=\left(\frac{w_{m}^{A}}{w_{m}^{B}}\right)^{1 /(4 \phi)-\phi} r_{m}^{A} .
$$

If $\gamma_{m}=4 / 3$, then $\phi=1 / 2$ and $r_{m}^{S}=r_{m}^{A}$, indicating that the jet is straight-sided as expected. Equations $(6.19 a, b)$ are useful because they can provide the change in radius, $r_{m}$, and change in velocity, $w_{m}^{S}$, between characteristic curves a priori. Although this analysis is based on the homogeneous system, knowledge of $w_{m}^{S}$ allows one to infer 
(a)

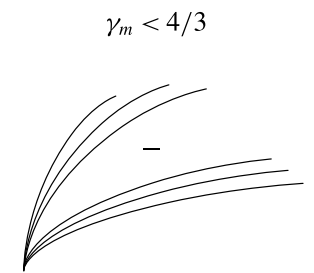

(b)

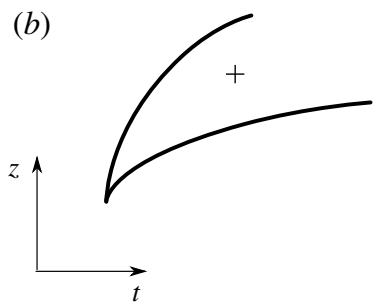

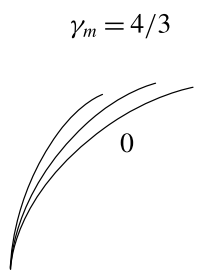

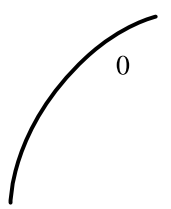

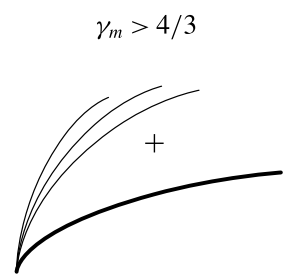

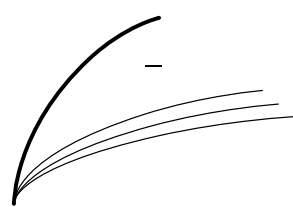

FIGURE 5. Response of system characteristic curves following a negative (step-down, $a$ ) and positive (step-up, $b$ ) change in the source momentum flux. A single bold line denotes a compression wave, three thin lines denote a rarefaction wave, and the symbols,,+- 0 denote a positive, negative and zero area change, respectively.

whether each characteristic curve represents a compression or rarefaction wave. This information is summarised in figure 5, which shows that although the nature of the leading wave is determined by the sign of the step change in $M_{m}$, it is the value of $\gamma_{m}$ that will determine the nature of the slower characteristic. Specifically, figures $6(a)$ and $6(b)$ provide contours of $w_{m}^{S} / w_{m}^{A}$ and $r_{m}^{S} / r_{m}^{A}$, respectively. The value of each of these variables in the region $S$ is determined by the relative change in source velocity $w_{m}^{A} / w_{m}^{B}$, and the profile parameter $\phi\left(\gamma_{m}\right)$ defined in (6.6). Whether the jet velocity or radius increases or decreases in the region $S$ is therefore indicated by the quadrant of figure $6(a, b)$ to which the pair $\left(\phi, w_{m}^{A} / w_{m}^{B}\right)$ belongs. The analysis performed in this section supports the inference that was made using the area equation (5.1) in $\S 5$. Namely, when $\gamma_{m}<4 / 3$ the radius of the jet increases or decreases in response to an increase or decrease in the source momentum flux, respectively, whilst for $\gamma_{m}>4 / 3$ the behaviour is reversed. Real jets, for which $\gamma_{m} \approx 4 / 3$, appear to occupy the special position between these states, in which the area is unaffected by changes in $M_{m}$.

\section{Conclusions}

In this paper we have used the momentum-energy framework described in Part 1 (Craske \& van Reeuwijk 2015) in a prognostic capacity to develop a model for unsteady jets that includes energy dispersion ((4.1) and (4.2)). The use of the mean energy equation allowed us to account for profile-related transport processes that are not evident at the level of momentum conservation. We applied Taylor's analysis of dispersion (Taylor 1953, 1954) to unsteady jets by considering how departures from a steady, self-similar state, relate to longitudinal gradients of integral quantities. By faithfully accounting for longitudinal transport processes the model is easily solved numerically using a central differencing scheme. For jets, our proposed model for type II dispersion appears to be more appropriate than the turbulence mixing term suggested by Scase \& Hewitt (2012) for plumes, the latter resulting in the nonphysical increase in a jet's momentum flux with height (see e.g. (2.18) or figure 1). 

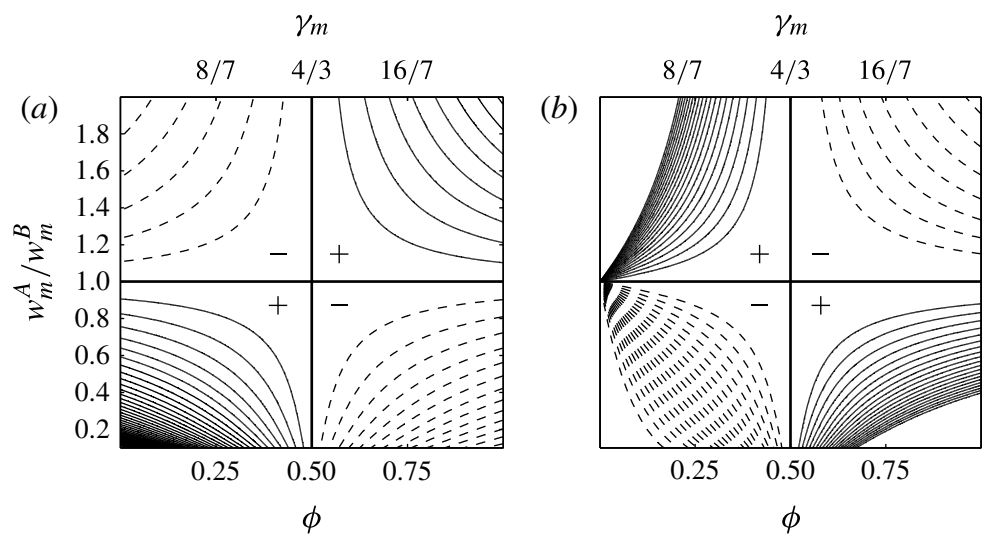

FIGURE 6. Response of relative jet velocity $w_{m}^{S} / w_{m}^{A}$ in $(a)$ and relative radius $r_{m}^{S} / r_{m}^{A}$ in $(b)$, in the region between characteristic curves for the homogeneous hyperbolic problem corresponding to (2.10) and (2.11) with constant $\gamma_{m}$ and $\delta_{m}=0$.

A comparison of the predictions obtained from the dispersion model with DNS results confirms that the closure provides a good representation of type II dispersion, and reproduces the location and longitudinal extent of fronts in the jets' momentum flux, over the parameter space investigated.

In addition, in $\S 6$ we determined the response of the jet to source perturbations, whose unbounded growth was found to be prevented by a sufficient amount of type I dispersion. The precise quantity of type I dispersion that causes the amplitude of perturbations to remain constant coincides with that obtained from a Gaussian velocity profile. Independently, in $\S 5$, we showed that Gaussian jets with a fixed source area remain straight-sided. We used this fact to develop a simplified version of our model equations. Both nonlinear and linear versions of the straight-sided model show a good agreement with the original model and the DNS data. Of interest in this respect is the connection with the model of steady plumes of Priestley \& Ball (1955), which, in contrast to the model of Morton et al. (1956), also predicts straight-sidedness. It is reasonable to expect that straight-sided solutions also exist for unsteady plumes, but that additional conditions involving the transport of buoyancy will also need to be satisfied. Interesting in this regard is how the growth of perturbations in an unsteady plume are affected by buoyancy and whether the value $\gamma_{m}=4 / 3$ is a distinguished case.

The problem considered in this paper consisted of an abrupt change in the source conditions of an established turbulent jet. Of further interest is the applicability of our findings to starting jets and plumes, in which quiescent fluid is displaced in front of an advancing region of turbulence. A characteristic feature of such flows is the existence of a turbulent/non-turbulent interface perpendicular to the longitudinal direction, which would most likely modify the dispersive viewpoint adopted here. In particular, one would expect the dimensionless longitudinal turbulent transport terms $\beta_{f}$ and $\gamma_{f}$ to play a significant role, and a local increase in $\delta_{m}$ to inhibit type II dispersion. An eddyviscosity-based closure for $\beta_{f}$ and $\gamma_{f}$ that modifies neither the steady-state jet solutions nor the steady-state plume solutions has the form

$$
\beta_{f} \propto \frac{1}{M_{m}^{1 / 2}} \frac{\partial Q_{m}}{\partial z}, \quad \gamma_{f}=2 \beta_{f},
$$


and can be incorporated into (4.1) and (4.2) without difficulty. Noteworthy in this regard, however, is the questionable use of an eddy-viscosity hypothesis to describe longitudinal turbulent fluxes such as $\overline{w^{\prime 2}}$. On the other hand, it is interesting that the lateral flux $\overline{u^{\prime} w^{\prime}}$, which ultimately determines the contribution from type II dispersion (see e.g. (3.7)), can be successfully described using an eddy-viscosity hypothesis (see e.g. Pope 2000).

\section{Acknowledgement}

J.C. gratefully acknowledges funding from the Engineering and Physical Sciences Research Council (EPSRC) under grant number EP/J500239/1.

\section{Appendix A. Jet similarity solutions}

In this section we re-derive the jet similarity solutions presented in Scase et al. (2006), without making an assumption about the velocity profile of the jet. Specifically, we seek solutions to unsteady transport equations for momentum (2.10) and mean energy (2.11):

$$
\begin{gathered}
\frac{\partial Q_{m}}{\partial t}+\frac{\partial M_{m}}{\partial z}=0 \\
\frac{\partial M_{m}}{\partial t}+\frac{\partial}{\partial z}\left(\gamma_{m} \frac{M_{m}^{2}}{Q_{m}}\right)=-2 \alpha_{0} \gamma_{m} \frac{M_{m}^{5 / 2}}{Q_{m}^{2}}
\end{gathered}
$$

of the form

$$
Q_{m}(z, t)=c_{1} z^{a_{1}} t^{b_{1}}, \quad M_{m}(z, t)=c_{2} z^{a_{2}} t^{b_{2}},
$$

for constants $a_{1}, a_{2}, b_{1}, b_{2}, c_{1}$ and $c_{2}$. In (A 2) we have related the dimensionless turbulence production $\delta_{m}$ to the steady-state entrainment coefficient according to $\delta_{m}=$ $-2 \alpha_{0} \gamma_{m}$. The solution to this problem is found to be

$$
Q_{m}(z, t)=\left(\frac{\gamma_{m} \alpha_{0}}{8-5 \gamma_{m}}\right)^{2} \frac{z^{3}}{t}, \quad M_{m}(z, t)=\frac{1}{4}\left(\frac{\gamma_{m} \alpha_{0}}{8-5 \gamma_{m}}\right)^{2} \frac{z^{4}}{t^{2}} .
$$

Using the definitions $r_{m} \equiv Q_{m} / M_{m}^{1 / 2}$ and $w_{m} \equiv M_{m} / Q_{m}$, one finds that the characteristic radius and velocity scale according to

$$
r_{m}(z)=\frac{2 \gamma_{m} \alpha_{0}}{8-5 \gamma_{m}} z, \quad w_{m}(z, t)=\frac{z}{4 t},
$$

respectively. Hence the behaviour of the velocity $w_{m}$ depends on neither the entrainment coefficient nor the shape of the radial profile of longitudinal velocity. The radius $r_{m}$, on the other hand, in spite of its time independence, is affected by both the entrainment coefficient and the velocity profile. For top-hat and Gaussian profiles the radius is given by

$$
\begin{gathered}
r_{m}\left(z, t \mid \gamma_{m}=1\right)=\frac{2 \alpha_{0} z}{3}, \\
r_{m}\left(z, t \mid \gamma_{m}=4 / 3\right)=2 \alpha_{0} z,
\end{gathered}
$$

respectively. Both the behaviour of $w_{m}$ in (A $\left.5 b\right)$ and that of $r_{m}$ for the top-hat profile (A 6) agree with the findings presented in Scase et al. (2006). Notably however, when 
the velocity profile is assumed to have a Gaussian form (A 7) shows that it retains its steady-state form, which is in agreement with the alternative derivation of straightsidedness presented in $\S 5$.

The form of $(\mathrm{A} 5 a, b)$ suggests that by redefining the entrainment coefficient, the similarity solutions can be represented in a form that is universally applicable to arbitrary velocity profiles. Recalling (2.12) and assuming that $\gamma_{m}$ is constant results in

$$
\alpha \equiv \underbrace{-\frac{\delta_{m}}{2 \gamma_{m}}}_{\alpha_{p r o d}}+\underbrace{\frac{Q_{m}}{M_{m}^{3 / 2}}\left(1-\frac{1}{\gamma_{m}}\right) \frac{\partial M_{m}}{\partial z}}_{\alpha_{d i s p 1}} .
$$

Therefore, using $\delta_{m}=-2 \gamma_{m} \alpha_{0}$ and the solutions (A $\left.4 a, b\right)$, we find that

$$
\alpha=\frac{3 \gamma_{m} \alpha_{0}}{8-5 \gamma_{m}}
$$

and we can therefore state that for all velocity profiles

$$
r_{m}(z, t)=\frac{2 \alpha z}{3} .
$$

Superficially, (A 10) has the same form as the top-hat solution (A 6), which was obtained by Scase et al. (2006). However, in general, in (A 10) $\alpha$ is not the same as the steady-state entrainment coefficient $\alpha_{0}$. Indeed, when $\gamma_{m}=4 / 3, \alpha=3 \alpha_{0}$, and the angle of spread of the jet is indistinguishable from its steady-state solution. More general solutions can be found that account for the dimensionless turbulent transport terms $\beta_{f}$ and $\gamma_{f}$.

\section{REFERENCES}

Abramowitz, M. \& Stegun, I. A. 1972 Handbook of Mathematical Functions: with Formulas, Graphs, and Mathematical Tables. Dover (eighth printing).

ARIS, R. 1956 On the dispersion of a solute in a fluid flowing through a tube. Proc. R. Soc. Lond. A 235 (1200), 67-77.

Chatwin, P. C. \& Allen, C. M. 1985 Mathematical models of dispersion in rivers and estuaries. Annu. Rev. Fluid Mech. 17 (1), 119-149.

Craske, J. \& VAn Reeuwijk, M. 2015 Energy dispersion in turbulent jets. Part 1. Direct simulation of steady and unsteady jets. J. Fluid Mech. 763, 500-537.

Delichatsios, M. A. 1979 Time similarity analysis of unsteady buoyant plumes in neutral surroundings. J. Fluid Mech. 93, 241-250.

FISCHER, H. B. 1973 Longitudinal dispersion and turbulent mixing in open-channel flow. Annu. Rev. Fluid Mech. 5 (1), 59-78.

Fischer, H. B., List, E. J., Koh, R. C. Y., Imberger, J. \& Brooks, N. 1979 Mixing in Inland and Coastal Waters. Academic.

Fox, D. G. 1970 Forced plume in a stratified fluid. J. Geophys. Res. 75 (33), 6818-6835.

Hunt, G. R. \& VAN DEN BREMER, T. S. 2011 Classical plume theory: 1937-2010 and beyond. IMA J. Appl. Maths 76 (3), 424-448.

KAminski, E., TAit, S. \& CARAZZO, G. 2005 Turbulent entrainment in jets with arbitrary buoyancy. J. Fluid Mech. 526, 361-376.

Kurganov, A. \& TADMOR, E. 2000 New high-resolution central schemes for nonlinear conservation laws and convection-diffusion equations. J. Comput. Phys. 160 (1), 241-282. 
Landel, J. R., Caulfield, C. P. \& Woods, A. W. 2012 Streamwise dispersion and mixing in quasi-two-dimensional steady turbulent jets. J. Fluid Mech. 711, 212-258.

Middleton, J. H. 1975 The asymptotic behaviour of a starting plume. J. Fluid Mech. 72, 753-771.

Morton, B. R., TAYlor, G. I. \& TURner, J. S. 1956 Turbulent gravitational convection from maintained and instantaneous sources. Proc. R. Soc. Lond. A 234 (1196), 1-23.

Panchapakesan, N. R. \& Lumley, J. L. 1993 Turbulence measurements in axisymmetric jets of air and helium. Part 1. Air jet. J. Fluid Mech. 246, 197-223.

Pope, S. B. 2000 Turbulent Flows. Cambridge University Press.

Priestley, C. H. B. \& BAll, F. K. 1955 Continuous convection from an isolated source of heat. Q. J. R. Meteorol. Soc. 81 (348), 144-157.

Ruban, A. I. \& Vonatsos, K. N. 2008 Discontinuous solutions of the boundary-layer equations. J. Fluid Mech. 614, 407-424.

Scase, M. M., Aspden, A. J. \& Caulfield, C. P. 2009 The effect of sudden source buoyancy flux increases on turbulent plumes. J. Fluid Mech. 635, 137-169.

Scase, M. M., Caulfield, C. P. \& Dalziel, S. B. 2008 Temporal variation of non-ideal plumes with sudden reductions in buoyancy flux. J. Fluid Mech. 600, 181-199.

Scase, M. M., Caulfield, C. P., Dalziel, S. B. \& Hunt, J. C. R. 2006 Time-dependent plumes and jets with decreasing source strengths. J. Fluid Mech. 563, 443-461.

SCASE, M. M. \& HewitT, R. E. 2012 Unsteady turbulent plume models. J. Fluid Mech. 697, 455-480.

TAYlor, G. I. 1953 Dispersion of soluble matter in solvent flowing slowly through a tube. Proc. R. Soc. Lond. A 219 (1137), 186-203.

TAYlor, G. I. 1954 The dispersion of matter in turbulent flow through a pipe. Proc. R. Soc. Lond. A 223 (1155), 446-468.

Toro, E. F. 1997 Riemann Solvers and Numerical Methods for Fluid Dynamics: a Practical Introduction. Springer.

Turner, J. S. 1962 The 'starting plume' in neutral surroundings. J. Fluid Mech. 13 (3), 356-368.

TURNER, J. S. 1986 Turbulent entrainment: the development of the entrainment assumption, and its application to geophysical flows. J. Fluid Mech. 173, 431-471.

Vul'Fson, A. N. \& BORODIn, O. O. 2001 Self-similar propagation regimes of a nonstationary high-temperature convective jet in the adiabatic atmosphere. J. Appl. Mech. Tech. Phys. 42, $255-261$.

YU, H. Z. 1990 Transient plume influence in measurement of convective heat release rates of fast-growing fires using a large-scale fire products collector. Trans. ASME: J. Heat Transfer 112 (1), 186-191. 